\title{
Neumann problems resonant at zero and infinity
}

\author{
Leszek Gasiński • Nikolaos S. Papageorgiou
}

Received: 8 December 2010 / Accepted: 20 January 2011 / Published online: 16 February 2011

(C) The Author(s) 2011. This article is published with open access at Springerlink.com

\begin{abstract}
We consider a semilinear Neumann problem with a reaction which is resonant at both zero and $\pm \infty$. Using a combination of methods from critical point theory, together with truncation techniques, the use of upper-lower solutions and of the Morse theory (critical groups), we show that the problem has at least five nontrivial smooth solutions, four of which have constant sign (two positive and two negative).
\end{abstract}

Keywords Resonance at zero and infinity - Critical point theory · Morse theory · Truncation techniques $\cdot$ Regularity theory $\cdot$ Multiple solutions $\cdot$ Solutions of constant sign

Mathematics Subject Classification (2000) $\quad 35 \mathrm{~J} 20 \cdot 35 \mathrm{~J} 60 \cdot 58 \mathrm{E} 05$

\section{Introduction}

Let $\Omega \subseteq \mathbb{R}^{N}$ be a bounded domain with a $C^{2}$-boundary $\partial \Omega$. In this paper, we study the following semilinear Neumann problem:

$$
\begin{cases}-\Delta u(z)=f(z, u(z)) & \text { in } \Omega \\ \frac{\partial u}{\partial n}=0 & \text { on } \partial \Omega .\end{cases}
$$

This research has been partially supported by the Ministry of Science and Higher Education of Poland under Grant no. N201 542438.

L. Gasiński ( $)$

Institute of Computer Science, Jagiellonian University, ul. Łojasiewicza 6, 30-348 Kraków, Poland e-mail: Leszek.Gasinski@ii.uj.edu.pl

\section{N. S. Papageorgiou}

Department of Mathematics, National Technical University, Zografou Campus,

15780 Athens, Greece

e-mail: npapg@math.ntua.gr 
Here, $f(z, \zeta)$ is a measurable function which is $C^{1}$ in the $\zeta$-variable. The aim of this work is to prove a multiplicity theorem when resonance occurs at both zero and $\pm \infty$. Such problems have been studied extensively in the context of Dirichlet equations. In this direction, we mention the works of Costa and Silva [8], Hirano and Nishimura [14], Landesman et al. [20], Liang and Su [22], Liu [24], Li and Su [25], Li and Zou [26], Su and Tang [34], Zou [37], and Zou and Liu [38]. For the corresponding Neumann problem, the bibliography is not that rich. There have been some existence and multiplicity results for resonant semilinear Neumann problems. We mention the works of Filippakis and Papageorgiou [10], Iannacci and Nkashama [15, 16], Kuo [19], Li [21], Li and Li [23], Mawhin [27], Mawhin et al. [28], Qian [33], and Tang and Wu [35]. Iannacci and Nkashama [15] and Kuo [19] use variants of the well-known Landesman-Lazer condition. Iannacci and Nkashama [16] use a sign condition, while Mawhin [27] and Mawhin et al. [28] use a monotonicity condition on the function $\zeta \longmapsto f(z, \zeta)$. All the aforementioned works prove existence theorems, but do not address the question of multiplicity of the nontrivial solutions. Multiplicity results can be found in the works of Filippakis and Papageorgiou [10], Li [21], Li and Li [23], Qian [33], and Tang and $\mathrm{Wu}$ [35]. In Li [21], $\mathrm{Li}$ and $\mathrm{Li}$ [23], and Qian [33], the authors deal with equations of the form

$$
\begin{cases}-\Delta u(z)+a u(z)=f(z, u(z)) & \text { in } \Omega, \\ \frac{\partial u}{\partial n}=0 & \text { on } \partial \Omega .\end{cases}
$$

In (1.2), the presence in the left-hand side of the term $a u$ (with $a>0$ ) facilitates the analysis of the equation, since in this case the differential operator in (1.2) is coercive. When $a=0$, this is no longer true (recall that the Poincaré inequality fails in the Sobolev space $H^{1}(\Omega)$ ). Li [21] and Li and Li [23] produce an infinity of nodal (i.e., sign changing) solutions, by assuming an oscillatory behavior for the reaction $f(z, \cdot)$. Their approach uses critical point theory, Leray-Schauder degree on order intervals, and Morse theory. Qian [33] deals with equations which are superlinear at $\pm \infty$, using the so-called Jeanjean condition. He produces a sequence of nodal solutions assuming a symmetry condition on $f(z, \cdot)$ (namely that $f(z, \cdot)$ is odd). His arguments are based on the critical point theory. Finally, Filippakis and Papageorgiou [10] and Tang and $\mathrm{Wu}[35]$ assume that $a=0$. Filippakis and Papageorgiou [10] permit the resonance at zero to be only with respect to the principal eigenvalue $\lambda_{0}=0$, impose a global sign condition on $f(z, \cdot)$, and produce only three nontrivial smooth solutions. Tang and $\mathrm{Wu}$ [35] employ an anticoercivity condition on the potential

$$
F(z, \zeta)=\int_{0}^{\zeta} f(z, s) \mathrm{d} s
$$

and using the local linking theorem (see e.g., [12, p. 665]), they establish the existence of two nontrivial solutions.

In this paper, using a combination of variational methods based on the critical point theory, with Morse theory (critical groups), we establish the existence of at least five nontrivial smooth solutions for problem (1.1), four of which have constant sign (two positive and two negative).

In the next section, for the convenience of the reader, we recall some of the main mathematical tools that we will use in the analysis of problem (1.1). 


\section{Mathematical background}

Let $X$ be a Banach space and let $X^{*}$ be its topological dual. By $\langle\cdot, \cdot\rangle$ we denote the duality brackets for the pair $\left(X^{*}, X\right)$. Let $\varphi \in C^{1}(X)$. We say that $\varphi$ satisfies the Cerami condition if the following is true:

"Every sequence $\left\{x_{n}\right\}_{n} \geqslant 1 \subseteq X$, such that

$$
\left|\varphi\left(x_{n}\right)\right| \leqslant M_{1} \text { and }\left(1+\left\|x_{n}\right\|\right) \varphi^{\prime}\left(x_{n}\right) \longrightarrow 0 \text { in } X^{*},
$$

for some $M_{1}>0$, admits a strongly convergent subsequence."

Using this compactness type condition, we can have the following minimax characterization of certain critical values of a $C^{1}$-functional. The result is known in the literature as the "mountain pass theorem".

Theorem 2.1 If $X$ is a Banach space, $\varphi \in C^{1}(X)$ satisfies the Cerami condition, $u_{0}, u_{1} \in X$ are such that $\left\|u_{0}-u_{1}\right\|>r>0$,

$$
\begin{gathered}
\max \left\{\varphi\left(u_{0}\right), \varphi\left(u_{1}\right)\right\}<\inf \left\{\varphi(u):\left\|u-u_{0}\right\|=r\right\}=\eta_{r}, \\
c=\inf _{\gamma \in \Gamma} \max _{0 \leqslant t \leqslant 1} \varphi(\gamma(t)),
\end{gathered}
$$

where

$$
\Gamma=\left\{\gamma \in C([0,1] ; X): \gamma(0)=u_{0}, \gamma(1)=u_{1}\right\},
$$

then $c \geqslant \eta_{r}$ and $c$ is a critical value of $\varphi$.

Throughout this work, we will use the following notation. Let $\varphi \in C^{1}(X)$ and let $c \in \mathbb{R}$. We set

$$
\begin{aligned}
\varphi^{c} & =\{u \in X: \varphi(u) \leqslant c\}, \\
K_{\varphi} & =\left\{u \in X: \varphi^{\prime}(u)=0\right\}, \\
K_{\varphi}^{c} & =\left\{u \in K_{\varphi}: \varphi(u)=c\right\} .
\end{aligned}
$$

Let $\left(Y_{1}, Y_{2}\right)$ be a topological pair and $Y_{1} \subseteq Y_{2} \subseteq X$. For every $k \geqslant 0$, by $H_{k}\left(Y_{2}, Y_{1}\right)$, we denote the $k$ th relative singular homology group with integer coefficients for the pair $\left(Y_{1}, Y_{2}\right)$. Recall that for all integers $k<0$, we have $H_{k}\left(Y_{2}, Y_{1}\right)=0$. The critical groups of $\varphi$ at an isolated critical point $u \in K_{\varphi}^{c}$ are defined by

$$
C_{k}(\varphi, u)=H_{k}\left(\varphi^{c} \cap U, \varphi^{c} \cap U \backslash\{u\}\right) \quad \forall k \geqslant 0,
$$

where $U$ is a neighborhood of $x$, such that

$$
K_{\varphi} \cap \varphi^{c} \cap U=\{u\}
$$

(see [7,29]). The excision property of singular homology theory implies that the above definition of critical groups is independent of the particular choice of the neighborhood $U$.

Suppose that $\varphi \in C^{1}(X)$ satisfies the Cerami condition and inf $\varphi\left(K_{\varphi}\right)>-\infty$. Choose $c<\inf \varphi\left(K_{\varphi}\right)$. The critical groups of $\varphi$ at infinity are defined by

$$
C_{k}(\varphi, \infty)=H_{k}\left(X, \varphi^{c}\right) \quad \forall k \geqslant 0
$$


(see [5]). The deformation theorem (see e.g., [12, p. 636]) implies that the above definition is independent of the choice of the particular level $c<\inf \varphi\left(K_{\varphi}\right)$. If $K_{\varphi}$ is finite, then we set

$$
\begin{aligned}
M(t, u) & =\sum_{k \geqslant 0} \operatorname{rank} C_{k}(\varphi, u) t^{k} \quad \forall t \in \mathbb{R}, u \in K_{\varphi}, \\
P(t, \infty) & =\sum_{k \geqslant 0} \operatorname{rank} C_{k}(\varphi, \infty) t^{k} \quad \forall t \in \mathbb{R} .
\end{aligned}
$$

Using these quantities, we have the Morse relation

$$
\sum_{u \in K_{\varphi}} M(t, u)=P(t, \infty)+(1+t) Q(t),
$$

where

$$
Q(t)=\sum_{k \geqslant 0} \beta_{k} t^{k}
$$

is a formal series in $t \in \mathbb{R}$ with nonnegative integer coefficients (see [7,29]).

Let $X=H$ be a Hilbert space, $u \in H, U$ a neighborhood of $u$ in $H$, and $\varphi \in C^{2}(U)$. If $u \in K_{\varphi}$, then the Morse index of $u$ is defined to be the supremum of the dimensions of the vector subspaces of $H$ on which $\varphi^{\prime \prime}(u)$ is negative definite. We say that $u \in K_{\varphi}$ is nondegenerate, if $\varphi^{\prime \prime}(u)$ is invertible. Suppose that $u \in K_{\varphi}$ is a nondegenerate critical point with Morse index $m$. Then

$$
C_{k}(\varphi, u)=\delta_{k, m} \mathbb{Z} \quad \forall k \geqslant 0,
$$

where

$$
\delta_{k, m}= \begin{cases}1 & \text { if } k=m \\ 0 & \text { if } k \neq m\end{cases}
$$

In the analysis of problem (1.1), we will use the following two "natural" spaces:

$$
C_{n}^{1}(\bar{\Omega})=\left\{u \in C^{1}(\bar{\Omega}): \frac{\partial u}{\partial n}(z)=0 \text { on } \partial \Omega\right\}
$$

(where $n(\cdot)$ denotes the outward unit normal on $\partial \Omega$ ) and

$$
H_{n}^{1}(\Omega)={\overline{C_{n}^{1}(\bar{\Omega})}}^{\|\cdot\|},
$$

where $\|\cdot\|$ denotes the usual Sobolev norm of $H^{1}(\Omega)$, i.e.,

$$
\|u\|^{2}=\|u\|_{2}^{2}+\|\nabla u\|_{2}^{2} \quad \forall u \in H^{1}(\Omega) .
$$

The space $C_{n}^{1}(\bar{\Omega})$ is an ordered Banach space, with positive cone

$$
C_{+}=\left\{u \in C_{n}^{1}(\bar{\Omega}): u(z) \geqslant 0 \text { for all } z \in \bar{\Omega}\right\} .
$$

This cone has a nonempty interior, given by

$$
\text { int } C_{+}=\left\{u \in C_{+}: u(z)>0 \text { for all } z \in \bar{\Omega}\right\} .
$$

For a large class of $C^{1}$-functionals, we can identify the $C_{n}^{1}(\bar{\Omega})$ and $H_{n}^{1}(\Omega)$ local minimizers. More precisely, let $g_{0}: \Omega \times \mathbb{R} \longrightarrow \mathbb{R}$ be a Carathéodory function, such that

$$
\left|g_{0}(z, \zeta)\right| \leqslant a_{0}(z)+c_{0}|\zeta|^{r-1} \text { for almost all } z \in \Omega \text {, all } \zeta \in \mathbb{R},
$$


with $a_{0} \in L^{\infty}(\Omega)_{+}, c_{0}>0$ and

$$
1<r<2^{*}= \begin{cases}\frac{2 N}{N-2} & \text { if } N \geqslant 3 \\ +\infty & \text { if } N=1,2\end{cases}
$$

(subcritical growth for $g_{0}(z, \cdot)$ ). We set

$$
G_{0}(z, \zeta)=\int_{0}^{\zeta} g_{0}(z, s) \mathrm{d} s
$$

and consider the $C^{1}$-functional $\psi_{0}: H_{n}^{1}(\Omega) \longrightarrow \mathbb{R}$, defined by

$$
\psi_{0}(u)=\frac{1}{2}\|\nabla u\|_{2}^{2}-\int_{\Omega} G_{0}(z, u(z)) \mathrm{d} z \quad \forall u \in H_{n}^{1}(\Omega) .
$$

Proposition 2.2 If $u_{0} \in H_{n}^{1}(\Omega)$ is a local $C_{n}^{1}(\bar{\Omega})$-minimizer of $\psi_{0}$, i.e., there exists $r_{0}>0$, such that

$$
\psi_{0}\left(u_{0}\right) \leqslant \psi_{0}\left(u_{0}+h\right) \quad \forall h \in C_{n}^{1}(\bar{\Omega}),\|h\|_{C_{n}^{1}(\bar{\Omega})} \leqslant r_{0},
$$

then $u_{0} \in C_{n}^{1}(\bar{\Omega})$ and it is a local $H_{n}^{1}(\Omega)$-minimizer of $\psi_{0}$, i.e., there exists $r_{1}>0$, such that

$$
\psi_{0}\left(u_{0}\right) \leqslant \psi_{0}\left(u_{0}+h\right) \quad \forall h \in H_{n}^{1}(\Omega),\|h\| \leqslant r_{1} .
$$

Remark 2.3 For the "Dirichlet" space $H_{0}^{1}(\Omega)$, this result was first proved by Brezis and Nirenberg [6] and was extended to the spaces $W_{0}^{1, p}(\Omega)$ (with $1<p<+\infty$ ) by Garcia Azorero et al. [11] (see also [13]). For the "Neumann" spaces $W_{n}^{1, p}(\Omega)(1<p<+\infty)$, the result can be found in Motreanu et al. [30] (for smooth functionals $\psi_{0}$ ) and in Iannizzotto and Papageorgiou [17] (for nonsmooth functionals $\psi_{0}$ ). A simplified proof of the result for more general operators than the $p$-Laplacian can be found in the recent work of Motreanu and Papageorgiou [31].

Next, we recall some basic facts about the spectrum of the negative Neumann Laplacian. So, let $m \in L^{\infty}(\Omega)_{+}, m \neq 0$ (a weight function), and consider the following weighted linear eigenvalue problem:

$$
\left\{\begin{array}{l}
-\Delta u(z)=\widehat{\lambda} m(z) u(z) \text { in } \Omega, \\
\frac{\partial u}{\partial n}=0 \text { on } \partial \Omega
\end{array}\right.
$$

Evidently a necessary condition for $\widehat{\lambda} \in \mathbb{R}$ to be an eigenvalue is that $\widehat{\lambda} \geqslant 0$. Moreover, $\widehat{\lambda}_{0}=\widehat{\lambda}_{0}(m)=0$ is an eigenvalue of $(2.2)$ with corresponding eigenspace $\mathbb{R}$ (the space of constant functions). Using the spectral theorem for compact operators, we can show that problem (2.2) has a sequence $\left\{\widehat{\lambda}_{k}(m)\right\}_{k \geqslant 0}$ of distinct eigenvalues, such that $\widehat{\lambda}_{k}(m) \longrightarrow+\infty$ as $k \rightarrow+\infty$. If $m \equiv 1$, we write $\widehat{\lambda}_{k}(1)=\widehat{\lambda}_{k}$ for all $k \geqslant 0$.

For every integer $k \geqslant 0$, by $E\left(\widehat{\lambda}_{k}(m)\right)$, we denote the eigenspace corresponding to the eigenvalue $\widehat{\lambda}_{k}(m)$. The regularity theory (see e.g., [12]) implies that $E\left(\widehat{\lambda}_{k}(m)\right) \subseteq C^{1}(\bar{\Omega})$. Moreover, we know that each $E\left(\widehat{\lambda}_{k}(m)\right)$ has the "unique continuation property", namely if $u \in E\left(\widehat{\lambda}_{k}(m)\right)$ vanishes on a set of positive measure, then $u(z)=0$ for all $z \in \bar{\Omega}$. We set

$$
\bar{H}_{i}=\bigoplus_{k=0}^{i} E\left(\widehat{\lambda}_{k}(m)\right) \text { and } \widehat{H}_{i}=\bar{H}_{i}^{\perp}=\overline{\bigoplus_{k \geqslant i+1} E\left(\widehat{\lambda}_{k}(m)\right)} .
$$


We have the following variational characterization for the eigenvalues $\widehat{\lambda}_{k}(m)$ :

$$
0=\widehat{\lambda}_{0}(m)=\min \left\{\frac{\|\nabla u\|_{2}^{2}}{\int_{\Omega} m u^{2} \mathrm{~d} z}: u \in H_{n}^{1}(\Omega), u \neq 0\right\}
$$

and for $k \geqslant 1$, we have

$$
\begin{aligned}
\widehat{\lambda}_{k}(m) & =\max \left\{\frac{\|\nabla \bar{u}\|_{2}^{2}}{\int_{\Omega} m \bar{u}^{2} \mathrm{~d} z}: \bar{u} \in \bar{H}_{k}, \bar{u} \neq 0\right\} \\
& =\min \left\{\frac{\|\nabla \widehat{u}\|_{2}^{2}}{\int_{\Omega} m \widehat{u}^{2} \mathrm{~d} z}: \widehat{u} \in \widehat{H}_{k}, \widehat{u} \neq 0\right\} .
\end{aligned}
$$

In (2.3), the minimum is attained on $E\left(\widehat{\lambda}_{k}(m)\right)=\mathbb{R}$, while in (2.4) the maximum and minimum are realized on $E\left(\widehat{\lambda}_{k}(m)\right), k \geqslant 1$.

As a consequence of these variational characterizations and of the unique continuation property, we have the following useful facts (see e.g., [10]).

Proposition 2.4 If $m, \widehat{m} \in L^{\infty}(\Omega)_{+} \backslash\{0\}, m(z) \leqslant \widehat{m}(z)$ for almost all $z \in \Omega$ and $m \neq \widehat{m}$, then

$$
\widehat{\lambda}_{k}(\widehat{m})<\widehat{\lambda}_{k}(m) \quad \forall k \geqslant 0 .
$$

Proposition 2.5 (a) If $k \geqslant-1$ is an integer, $\eta \in L^{\infty}(\Omega)_{+}, \eta(z) \leqslant \widehat{\lambda}_{k+1}$ for almost all $z \in \Omega$ and $\eta \neq \widehat{\lambda}_{k+1}$, then there exists $\xi_{0}>0$, such that

$$
\|\nabla \widehat{u}\|_{2}^{2}-\int_{\Omega} \eta \widehat{u}^{2} d z \geqslant \xi_{0}\|\widehat{u}\|^{2} \quad \forall \widehat{u} \in \widehat{H}_{k} .
$$

(b) If $k \geqslant 0$ is an integer, $\eta \in L^{\infty}(\Omega)_{+}, \eta(z) \geqslant \widehat{\lambda}_{k}$ for almost all $z \in \Omega$ and $\eta \neq \widehat{\lambda}_{k}$, then there exists $\xi_{1}>0$, such that

$$
\|\nabla \bar{u}\|_{2}^{2}-\int_{\Omega} \eta \bar{u}^{2} d z \leqslant-\xi_{1}\|\bar{u}\|^{2} \quad \forall \bar{u} \in \bar{H}_{k} .
$$

From the eigenvalues $\left\{\widehat{\lambda}_{k}(m)\right\}_{k \geqslant 0}$ only the first one $\widehat{\lambda}_{0}(m)=0$ has constant sign eigenfunction. All the other eigenvalues have nodal (i.e., sign changing) eigenfunctions. In what follows $\widehat{u}_{0}$ denotes the $L^{2}$-normalized, positive principal eigenfunction, i.e., $\widehat{u}_{0}=\frac{1}{|\Omega|_{N}^{\frac{1}{2}}}$ (hereafter, by $|\cdot|_{N}$ we denote the Lebesgue measure on $\mathbb{R}^{N}$ ).

The next result, due to Liang and Su [22] (see also [18] for an extension to Banach spaces), is helpful in computing critical groups. It is a generalization of an earlier result of Perera and Schechter [32].

Proposition 2.6 If $H$ is a Hilbert space, $\left\{h_{t}\right\}_{t \in[0,1]} \subseteq C^{1}(H)$ is a family of functionals, such that $\left(h_{t}\right)^{\prime}$ and $\partial_{t} h_{t}$ are both locally Lipschitz, $h_{0}$ and $h_{1}$ satisfy the Cerami condition and there exist $a \in \mathbb{R}$ and $\delta>0$, such that

$$
h_{t}(u) \leqslant a \Longrightarrow\left((1+\|u\|)\left\|h_{t}^{\prime}(u)\right\|_{*} \geqslant \delta \text { for all } t \in[0,1]\right),
$$

then

$$
C_{k}\left(h_{0}, \infty\right)=C_{k}\left(h_{1}, \infty\right) \quad \forall k \geqslant 0 .
$$


Remark 2.7 Note that, if there exists $R>0$, such that

$$
\inf \left\{(1+\|u\|)\left\|h_{t}^{\prime}(u)\right\|_{*}: t \in[0,1],\|u\|>R\right\}>0
$$

and

$$
\inf \left\{h_{t}(u): t \in[0,1],\|u\| \leqslant R\right\}>-\infty
$$

then (2.5) holds.

In the sequel, we will use the notation $r^{ \pm}=\max \{ \pm r, 0\}$ for all $r \in \mathbb{R}$. Also, by $\|\cdot\|$ we denote the norm of the Sobolev space $H^{1}(\Omega)$ and by $|\cdot|_{N}$ the Lebesgue measure on $\mathbb{R}^{N}$. Finally by $\|\cdot\|_{p}(1<p<\infty)$, we denote the norm of $L^{p}(\Omega)$ of $L^{p}(\Omega ; \mathbb{R})$ and $p^{\prime}>1$ is the conjugate exponent of $p>1$, i.e., $\frac{1}{p}+\frac{1}{p^{\prime}}=1$.

\section{The Cerami condition}

The hypotheses on the reaction term $f$ are the following:

$H_{f}: f: \Omega \times \mathbb{R} \longrightarrow \mathbb{R}$ is a measurable function, such that for almost all $z \in \Omega$, we have $f\left(\overline{z, 0)}=0, f(z, \cdot) \in C^{1}(\mathbb{R})\right.$ and

(i) $\left|f_{\zeta}^{\prime}(z, \zeta)\right| \leqslant a(z)+c|\zeta|^{r-2}$ for almost all $z \in \Omega$, all $\zeta \in \mathbb{R}$ with $a \in L^{\infty}(\Omega)_{+}, c>0$ and $2 \leqslant r \leqslant 2^{*}$

(ii) there exist integer $i \geqslant 1, \alpha \in(0,1)$, and $\eta_{\infty} \in L^{\infty}(\Omega)$ with $\eta_{\infty}(z) \leqslant 0$ for almost all $z \in \Omega, \eta_{\infty} \neq 0$, such that

$$
\lim _{|\zeta| \rightarrow+\infty} \frac{f(z, \zeta)}{\zeta}=\widehat{\lambda}_{i}
$$

uniformly for almost all $z \in \Omega$ and, if $f_{\infty}(z, \zeta)=f(z, \zeta)-\widehat{\lambda}_{i} \zeta$, then

$$
\lim _{|\zeta| \rightarrow+\infty} \frac{f_{\infty}(z, \zeta)}{|\zeta|^{\alpha}}=0 \text { and } \limsup _{|\zeta| \rightarrow+\infty} \frac{f_{\infty}(z, \zeta) \zeta}{|\zeta|^{2 \alpha}} \leqslant \eta_{\infty}(z)
$$

uniformly for almost all $z \in \Omega$;

(iii) there exist integer $m \geqslant 1, m \neq i, \beta>1$, and $\eta_{0} \in L^{\infty}(\Omega)$ with $\eta_{0}(z) \leqslant 0$ for almost all $z \in \Omega, \eta_{0} \neq 0$, such that

$$
f_{\zeta}^{\prime}(z, 0)=\lim _{\zeta \rightarrow 0} \frac{f(z, \zeta)}{\zeta}=\widehat{\lambda}_{m}
$$

uniformly for almost all $z \in \Omega$ and if $f_{0}(z, \zeta)=f(z, \zeta)-\widehat{\lambda}_{m} \zeta$, then

$$
\lim _{\zeta \rightarrow 0} \frac{f_{0}(z, \zeta)}{|\zeta|^{\beta}}=0 \text { and } \limsup _{\zeta \rightarrow 0} \frac{f_{0}(z, \zeta) \zeta}{|\zeta|^{2 \beta}} \leqslant \eta_{0}(z)
$$

uniformly for almost all $z \in \Omega$;

(iv) there exist numbers $a_{-}<0<a_{+}$, such that

$$
f\left(z, a_{+}\right) \leqslant 0 \leqslant f\left(z, a_{-}\right) \text {for almost all } z \in \Omega
$$

and $f\left(\cdot, a_{-}\right) \neq 0, f\left(\cdot, a_{+}\right) \neq 0$. 
Remark 3.1 Hypothesis $H_{f}$ (ii) implies that the problem is resonant at infinity, while hypothesis $H_{f}$ (iii) implies that the problem is resonant at zero. So, we have a kind of "double resonance".

Example 3.2 The following function $f$ satisfies hypotheses $H_{f}$ (for the sake of simplicity we drop the $z$-dependence):

$$
f(\zeta)= \begin{cases}\widehat{\lambda}_{m} \zeta-\xi|\zeta|^{r-2} \zeta & \text { if }|\zeta| \leqslant 1 \\ \widehat{\lambda}_{i} \zeta-\hat{\xi}|\zeta|^{q-2} \zeta & \text { if }|\zeta|>1\end{cases}
$$

with $1<q<2<r<+\infty, \xi>\widehat{\lambda}_{m}$ and $\widehat{\xi}=\xi+\widehat{\lambda}_{i}-\widehat{\lambda}_{m}>0$. For this example, we take $\alpha \in\left(q-1, \frac{q}{2}\right), \beta \in\left(\frac{r}{2}, r-1\right), a_{-}=-1$, and $a_{+}=1$.

Let $\varphi: H_{n}^{1}(\Omega) \longrightarrow \mathbb{R}$ be the energy functional for problem (1.1), defined by

$$
\varphi(u)=\frac{1}{2}\|\nabla u\|_{2}^{2}-\int_{\Omega} F(z, u(z)) \mathrm{d} z \quad \forall u \in H_{n}^{1}(\Omega) .
$$

We know that $\varphi \in C^{2}\left(H_{n}^{1}(\Omega)\right)$. Moreover

$$
\varphi^{\prime}(u)=A(u)-N_{f}(u) \quad \forall u \in H_{n}^{1}(\Omega),
$$

where $A \in \mathcal{L}\left(H_{n}^{1}(\Omega), H_{n}^{1}(\Omega)^{*}\right)$ is defined by

$$
\langle A(u), y\rangle=\int_{\Omega}(\nabla u, \nabla y)_{\mathbb{R}^{N}} \mathrm{~d} z \quad \forall u, y \in H_{n}^{1}(\Omega)
$$

and $N_{f}(y)(\cdot)=f(\cdot, y(\cdot))$ for all $y \in H_{n}^{1}(\Omega)$. Also

$$
\left\langle\varphi^{\prime \prime}(u) y, v\right\rangle=\int_{\Omega}(\nabla y, \nabla v)_{\mathbb{R}^{N}} \mathrm{~d} z-\int_{\Omega} f_{\zeta}^{\prime}(z, u(z)) y v \mathrm{~d} z \quad \forall u, y, v \in H_{n}^{1}(\Omega) .
$$

Note that $\varphi^{\prime \prime}(u) \in \mathcal{L}\left(H_{n}^{1}(\Omega), H_{n}^{1}(\Omega)^{*}\right)$ is a Fredholm operator.

Using the eigenvalue $\widehat{\lambda}_{i}>0$, we can have the following orthogonal direct sum decomposition of the Sobolev space $H_{n}^{1}(\Omega)$ :

$$
\left.H_{n}^{1}(\Omega)=\bar{H}_{i-1} \oplus E \widehat{\lambda}_{i}\right) \oplus \widehat{H}_{i+1},
$$

where

$$
\bar{H}_{i-1}=\bigoplus_{k=0}^{i-1} E\left(\widehat{\lambda}_{k}\right) \text { and } \widehat{H}_{i+1}=\overline{\bigoplus_{k \geqslant i+1} E\left(\widehat{\lambda}_{k}\right)}
$$

Then for every $u \in H_{n}^{1}(\Omega)$, we have

$$
u=\bar{u}+u^{0}+\widehat{u},
$$

with $\bar{u} \in \bar{H}_{i-1}, u^{0} \in E\left(\widehat{\lambda}_{i}\right), \widehat{u} \in \widehat{H}_{i+1}$. This decomposition is unique.

Proposition 3.3 If $\left\{u_{n}\right\}_{n} \geqslant 1 \subseteq H_{n}^{1}(\Omega)$ is a sequence, such that

$$
\left\|u_{n}\right\| \longrightarrow+\infty \text { and } \frac{\widehat{u}_{n}+\bar{u}_{n}}{\left\|u_{n}\right\|} \longrightarrow 0 \text { in } H_{n}^{1}(\Omega),
$$


then

$$
\limsup _{n \rightarrow+\infty} \int_{\Omega} \frac{f_{\infty}\left(z, u_{n}\right) u_{n}}{\left\|u_{n}\right\|^{2 \alpha}} d z<0
$$

Proof From Bartolo et al. [3], we know that for a given $\varepsilon>0$, we can find $m_{1}(\varepsilon)>0$ small enough and $m_{2}(\varepsilon)>0$ large enough, such that

$$
\left|\left\{z \in \Omega:\left|u^{0}(z)\right|<m_{1}\left\|u^{0}\right\|\right\}\right|_{N}<\varepsilon \quad \forall u^{0} \in E\left(\widehat{\lambda}_{i}\right)
$$

and

$$
\begin{aligned}
& \left|\left\{z \in \Omega:|\widehat{u}(z)+\bar{u}(z)|>m_{2}\|\widehat{u}+\bar{u}\|\right\}\right|_{N} \\
& \quad<m_{1}^{\frac{2 \alpha}{1-\alpha}} \varepsilon^{\frac{1}{1-\alpha}} \leqslant \varepsilon \quad \forall \widehat{u} \in \widehat{H}_{i+1}, \bar{u} \in \bar{H}_{i-1} .
\end{aligned}
$$

For every $n \geqslant 1$, we introduce the following sets

$$
\begin{aligned}
& D_{1 n}=\left\{z \in \Omega:\left|u^{0}(z)\right| \geqslant m_{1}\left\|u^{0}\right\|\right\}, \\
& D_{2 n}=\left\{z \in \Omega:|\widehat{u}(z)+\bar{u}(z)| \leqslant m_{2}\|\widehat{u}+\bar{u}\|\right\} .
\end{aligned}
$$

From (3.1) and (3.2), it follows that

$$
\left|\Omega \backslash D_{1 n}\right|_{N}<\varepsilon, \quad\left|\Omega \backslash D_{2 n}\right|_{N}<\varepsilon
$$

and

$$
\left|D_{1 n} \cap D_{2 n}\right|_{N} \geqslant\left|\Omega_{1}\right|_{N}-\left|\Omega \backslash D_{2 n}\right|_{N} \geqslant|\Omega|_{N}-2 \varepsilon .
$$

Choosing $\varepsilon \in\left(0, \frac{1}{2}|\Omega|_{N}\right)$, we see that

$$
\left|D_{1 n} \cap D_{2 n}\right|>0
$$

and so $D_{1 n} \cap D_{2 n} \neq \emptyset$. Let $z \in D_{1 n} \cap D_{2 n}$. Then

$$
\begin{aligned}
\frac{\left|u_{n}(z)\right|}{\left\|u_{n}\right\|} & =\frac{\left|u_{n}^{0}(z)+\widehat{u}_{n}(z)+\bar{u}_{n}(z)\right|}{\left\|u_{n}\right\|} \geqslant \frac{\left|u_{n}^{0}(z)\right|}{\left\|u_{n}\right\|}-\frac{\left|\widehat{u}_{n}(z)+\bar{u}_{n}(z)\right|}{\left\|u_{n}\right\|} \\
& \geqslant \frac{m_{1}\left\|u_{n}^{0}\right\|}{\left\|u_{n}\right\|}-\frac{m_{2}\left\|\widehat{u}_{n}+\bar{u}_{n}\right\|}{\left\|u_{n}\right\|} .
\end{aligned}
$$

Next let $z \in D_{2 n} \backslash D_{1 n}$. Then

$$
\begin{aligned}
\frac{\left|u_{n}(z)\right|}{\left\|u_{n}\right\|} & =\frac{\left|u_{n}^{0}(z)+\widehat{u}_{n}(z)+\bar{u}_{n}(z)\right|}{\left\|u_{n}\right\|} \leqslant \frac{\left|u_{n}^{0}(z)\right|}{\left\|u_{n}\right\|}-\frac{\left|\widehat{u}_{n}(z)+\bar{u}_{n}(z)\right|}{\left\|u_{n}\right\|} \\
& <\frac{m_{1}\left\|u_{n}^{0}\right\|}{\left\|u_{n}\right\|}-\frac{m_{2}\left\|\widehat{u}_{n}+\bar{u}_{n}\right\|}{\left\|u_{n}\right\|} .
\end{aligned}
$$

By virtue of hypotheses $H_{f}(i)$ and $(i i)$, we can find $c_{1}=c_{1}(\varepsilon)>0$, such that

$$
f_{\infty}(z, \zeta) \zeta \leqslant\left(\eta_{\infty}(z)+m_{1}^{2 \alpha} \varepsilon\right)|\zeta|^{2 \alpha}+c_{1} \text { for almost all } z \in \Omega, \text { all } \zeta \in \mathbb{R} \text {. }
$$


Then, using (3.6), we have

$$
\begin{aligned}
& \int_{D_{1 n} \cap D_{2 n}} \frac{f_{\infty}\left(z, u_{n}\right) u_{n}}{\left\|u_{n}\right\|^{2 \alpha}} \mathrm{d} z \\
& \leqslant \int_{D_{1 n} \cap D_{2 n}}\left(\eta_{\infty}(z)+\varepsilon\right)\left(\frac{\left|u_{n}\right|}{\left\|u_{n}\right\|}\right)^{2 \alpha} \mathrm{d} z+\frac{c_{1}}{\left\|u_{n}\right\|^{2 \alpha}}|\Omega|_{N} \\
& \leqslant m_{1}^{2 \alpha}\left(\frac{\left\|u_{n}^{0}\right\|}{\left\|u_{n}\right\|}\right)^{2 \alpha} \int_{D_{1 n} \cap D_{2 n}} \eta_{\infty}(z) \mathrm{d} z \\
& -m_{2}^{2 \alpha}\left(\frac{\left\|\widehat{u}_{n}+\bar{u}_{n}\right\|}{\left\|u_{n}\right\|}\right)^{2 \alpha} \int_{D_{1 n} \cap D_{2 n}} \eta_{\infty}(z) \mathrm{d} z \\
& +\varepsilon \int_{D_{1 n} \cap D_{2 n}}\left(\frac{\left|u_{n}\right|}{\left\|u_{n}\right\|}\right)^{2 \alpha} \mathrm{d} z+\frac{c_{1}}{\left\|u_{n}\right\|^{2 \alpha}}|\Omega|_{N}
\end{aligned}
$$

(since $\eta_{\infty} \leqslant 0$; see $\left.H_{f}(i i)\right)$. Also, hypotheses $H_{f}(i)$ and $(i i)$, imply that

$$
\left|f_{\infty}(z, \zeta) \zeta\right| \leqslant c_{2}\left(|\zeta|^{2 \alpha}+1\right) \text { for almost all } z \in \Omega \text {, all } \zeta \in \mathbb{R}
$$

with $c_{2}>0$. Hence, using (3.8), we have

$$
\begin{aligned}
& \int_{D_{2 n} \backslash D_{1 n}} \frac{f_{\infty}\left(z, u_{n}\right) u_{n}}{\left\|u_{n}\right\|^{2 \alpha}} \mathrm{d} z \\
& \leqslant c_{2} \int_{D_{2 n} \backslash D_{1 n}}\left(\frac{\left|u_{n}\right|}{\left\|u_{n}\right\|}\right)^{2 \alpha} \mathrm{d} z+\frac{c_{2}}{\left\|u_{n}\right\|^{2 \alpha}}|\Omega|_{N} \\
& \leqslant c_{2} m_{1}^{2 \alpha}\left(\frac{\left\|u_{n}^{0}\right\|}{\left\|u_{n}\right\|}\right)^{2 \alpha} \varepsilon+c_{2} m_{2}^{2 \alpha}\left(\frac{\left\|\widehat{u}_{n}+\bar{u}_{n}\right\|}{\left\|u_{n}\right\|}\right)^{2 \alpha}|\Omega|_{N}+\frac{c_{2}}{\left\|u_{n}\right\|^{2 \alpha}}|\Omega|_{N}
\end{aligned}
$$

(see (3.5) and (3.3)). Moreover, we have

$$
\int_{\Omega \backslash D_{2 n}} \frac{f_{\infty}\left(z, u_{n}\right) u_{n}}{\left\|u_{n}\right\|^{2 \alpha}} \mathrm{d} z \leqslant c_{2} \int_{\Omega \backslash D_{2 n}}\left(\frac{\left|u_{n}\right|}{\left\|u_{n}\right\|}\right)^{2 \alpha} \mathrm{d} z+\frac{c_{2}}{\left\|u_{n}\right\|^{2 \alpha}}|\Omega|_{N}
$$

(see (3.8)). Note that $\left(\frac{u_{n}(\cdot)}{\left\|u_{n}\right\|}\right)^{2 \alpha} \in L^{\frac{1}{\alpha}}(\Omega)$. So, by virtue of Hölder inequality and (3.3), we have

$$
\begin{aligned}
\int_{\Omega \backslash D_{2 n}}\left(\frac{\left|u_{n}\right|}{\left\|u_{n}\right\|}\right)^{2 \alpha} \mathrm{d} z & =\int_{\Omega} \chi_{\Omega \backslash D_{2 n}}\left(\frac{\left|u_{n}\right|}{\left\|u_{n}\right\|}\right)^{2 \alpha} \mathrm{d} z \\
& \leqslant\left(\frac{\left\|u_{n}\right\|_{2}}{\left\|u_{n}\right\|}\right)^{2 \alpha}\left|\Omega \backslash D_{2 n}\right|_{N}^{1-\alpha} \leqslant m_{1}^{2 \alpha} \varepsilon .
\end{aligned}
$$


therefore, finally we have

$$
\begin{aligned}
\int_{\Omega} & \frac{f_{\infty}\left(z, u_{n}\right) u_{n}}{\left\|u_{n}\right\|^{2 \alpha}} \mathrm{d} z \\
= & \int_{D_{1 n} \cap D_{2 n}} \frac{f_{\infty}\left(z, u_{n}\right) u_{n}}{\left\|u_{n}\right\|^{2 \alpha}} \mathrm{d} z+\int_{D_{2 n} \backslash D_{1 n}} \frac{f_{\infty}\left(z, u_{n}\right) u_{n}}{\left\|u_{n}\right\|^{2 \alpha}} \mathrm{d} z \\
& +\int_{\Omega \backslash D_{2 n}} \frac{f_{\infty}\left(z, u_{n}\right) u_{n}}{\left\|u_{n}\right\|^{2 \alpha}} \mathrm{d} z \\
\leqslant & m_{1}^{2 \alpha}\left(\frac{\left\|u_{n}^{0}\right\|}{\left\|u_{n}\right\|}\right)^{2 \alpha} \int_{D_{1 n} \cap D_{2 n}} \eta_{\infty}(z) \mathrm{d} z+m_{2}^{2 \alpha}\left(\frac{\left\|\widehat{u}_{n}+\bar{u}_{n}\right\|}{\left\|u_{n}\right\|}\right)^{2 \alpha}\left\|\eta_{\infty}\right\|_{1} \\
& +m_{1}^{2 \alpha} \varepsilon \int_{D_{1 n} \cap D_{2 n}}\left(\frac{\left|u_{n}\right|}{\left\|u_{n}\right\|}\right)^{2 \alpha} \mathrm{d} z+\frac{c_{1}}{\left\|u_{n}\right\|^{2 \alpha}}|\Omega|_{N} \\
& +c_{2} m_{1}^{2 \alpha}\left(\frac{\left\|u_{n}^{0}\right\|}{\left\|u_{n}\right\|}\right)^{2 \alpha} \varepsilon+c_{2} m_{2}^{2 \alpha}\left(\frac{\left\|\widehat{u}_{n}+\bar{u}_{n}\right\|}{\left\|u_{n}\right\|}\right)^{2 \alpha}|\Omega|_{N} \\
& +\frac{c_{2}}{\left\|u_{n}\right\|^{2 \alpha}}|\Omega|_{N}+m_{1}^{2 \alpha} \varepsilon
\end{aligned}
$$

(see (3.7), (3.9) and (3.10)). Note that

$$
\chi_{D_{1 n} \cap D_{2 n}}(z) \longrightarrow \chi_{\Omega}(z)=1 \text { almost everywhere on } \Omega \text { as } \varepsilon \searrow 0
$$

(see (3.3)). So, if in (3.11) we pass to the limit as $n \rightarrow+\infty$, we have

$$
\begin{aligned}
& \int_{\Omega} \frac{f_{\infty}\left(z, u_{n}\right) u_{n}}{\left\|u_{n}\right\|^{2 \alpha}} \mathrm{d} z \\
& \leqslant m_{1}^{2 \alpha}\left(\frac{\left\|u_{n}^{0}\right\|}{\left\|u_{n}\right\|}\right)^{2 \alpha} \int_{\Omega} \eta_{\infty}(z) \mathrm{d} z+m_{2}^{2 \alpha}\left(\frac{\left\|\bar{u}_{n}+\widehat{u}_{n}\right\|}{\left\|u_{n}\right\|}\right)^{2 \alpha}\left\|\eta_{\infty}\right\|_{1} \\
& \quad+\frac{c_{1}}{\left\|u_{n}\right\|^{2 \alpha}}|\Omega|_{N}+c_{2} m_{2}^{2 \alpha}\left(\frac{\left\|\widehat{u}_{n}+\bar{u}_{n}\right\|}{\left\|u_{n}\right\|}\right)^{2 \alpha}|\Omega|_{N}+\frac{c_{2}}{\left\|u_{n}\right\|^{2 \alpha}}|\Omega|_{N},
\end{aligned}
$$

so

$$
\limsup _{n \rightarrow+\infty} \int_{\Omega} \frac{f_{\infty}\left(z, u_{n}\right) u_{n}}{\left\|u_{n}\right\|^{2 \alpha}} \mathrm{d} z \leqslant m_{1}^{2 \alpha}\left(\int_{\Omega} \eta_{\infty}(z) \mathrm{d} z+\varepsilon(\widehat{c}+1)\right)
$$

for some $\widehat{c}>0$. (recall that $\frac{\widehat{u}_{n}+\bar{u}_{n}}{\left\|u_{n}\right\|} \longrightarrow 0$ in $H_{n}^{1}(\Omega)$ ). Let us choose $\varepsilon<-\frac{1}{\widehat{c}+1} \int_{\Omega} \eta_{\infty}(z) \mathrm{d} z$ to finish the proof.

For $R>0$ and $\vartheta \in(0,1)$, we introduce the set

$$
C_{\infty}(R, \vartheta, \alpha)=\left\{u \in H_{n}^{1}(\Omega):\|u\| \geqslant R,\|\widehat{u}+\bar{u}\| \leqslant \vartheta\|u\|^{\alpha}\right\} .
$$

Proposition 3.4 If hypotheses $H_{f}$ (i) and (ii) hold, then there exist $R>0, \vartheta \in(0,1)$ and $\widehat{\delta}>0$, such that

$$
\left\langle\varphi^{\prime}\left(u_{0}\right), u^{0}\right\rangle \geqslant \widehat{\delta} \forall u \in C_{\infty}(R, \vartheta, \alpha)
$$


Proof We argue indirectly. So, suppose that the proposition is not true. Then for any $\vartheta=$ $\widehat{\delta}=\frac{1}{n}, n \geqslant 1$, we can find $u_{n} \in H_{n}^{1}(\Omega)$, such that

$$
\left\|u_{n}\right\| \geqslant n, \quad\left\|\widehat{u}_{n}+\bar{u}_{n}\right\| \leqslant \frac{1}{n}\left\|u_{n}\right\|^{\alpha} \quad \text { and } \quad\left\langle\varphi^{\prime}\left(u_{n}\right), u_{n}^{0}\right\rangle<\frac{1}{n} \quad \forall n \geqslant 1 .
$$

From (3.12), we see that

$$
\left\|u_{n}\right\| \longrightarrow+\infty, \frac{\left\|\widehat{u}_{n}+\bar{u}_{n}\right\|}{\left\|u_{n}\right\|^{\alpha}} \longrightarrow 0
$$

and

$$
\left\langle\varphi^{\prime}\left(u_{n}\right), u_{n}^{0}\right\rangle=-\int_{\Omega} f_{\infty}\left(z, u_{n}\right) u_{n}^{0} \mathrm{~d} z<\frac{1}{n}
$$

(since $\left\|\nabla u_{n}^{0}\right\|_{2}^{2}=\widehat{\lambda}_{i}\left\|u_{n}^{0}\right\|_{2}^{2}$ for all $n \geqslant 1$ ). From (3.14), it follows that

$$
\liminf _{n \geqslant+\infty} \int_{\Omega} \frac{f_{\infty}\left(z, u_{n}\right) u_{n}^{0}}{\left\|u_{n}\right\|^{2 \alpha}} \mathrm{d} z \geqslant 0
$$

On the other hand, by virtue of hypotheses $H_{f}(i)$ and $(i i)$, for a given $\varepsilon>0$, we can find $c_{3}=c_{3}(\varepsilon)>0$, such that

$$
\left|f_{\infty}(z, \zeta)\right| \leqslant \varepsilon|\zeta|^{\alpha}+c_{3} \text { for almost all } z \in \Omega, \text { all } \zeta \in \mathbb{R} .
$$

Then, using (3.16), we have

$$
\begin{aligned}
& \left|\int_{\Omega} \frac{f_{\infty}\left(z, u_{n}\right)\left(\widehat{u}_{n}+\bar{u}_{n}\right)}{\left\|u_{n}\right\|^{2 \alpha}} \mathrm{d} z\right| \\
& \quad \leqslant \int_{\Omega} \frac{\left(\varepsilon\left|u_{n}\right|^{\alpha}+c_{3}\right)\left|\widehat{u}_{n}+\bar{u}_{n}\right|}{\left\|u_{n}\right\|^{2 \alpha}} \mathrm{d} z \\
& \quad \leqslant \varepsilon \int_{\Omega}\left(\frac{\left|u_{n}\right|}{\left\|u_{n}\right\|}\right)^{\alpha} \frac{|\widehat{u}+\bar{u}|}{\left\|u_{n}\right\|^{\alpha}} \mathrm{d} z+c_{4} \frac{\left\|\widehat{u}_{n}+\bar{u}_{n}\right\|}{\left\|u_{n}\right\|^{2 \alpha}} \quad \forall n \geqslant 1,
\end{aligned}
$$

for some $c_{4}>0$. Note that

$$
\left(\frac{u_{n}(\cdot)}{\left\|u_{n}\right\|}\right)^{\alpha} \in L^{\frac{2}{\alpha}}(\Omega) \text { and }\left(\frac{2}{\alpha}\right)^{\prime}=\frac{2}{2-\alpha} \leqslant 2^{*}=\frac{2 N}{N-2} \text { if } N \geqslant 3 .
$$

Hence, $\widehat{u}_{n}+\bar{u}_{n} \in L^{\frac{2}{2-\alpha}}(\Omega)$ and we can apply Hölder inequality and obtain

$$
\int_{\Omega}\left(\frac{\left|u_{n}\right|}{\left\|u_{n}\right\|}\right)^{\alpha} \frac{\left|\widehat{u}_{n}+\bar{u}_{n}\right|}{\left\|u_{n}\right\|^{\alpha}} \mathrm{d} z \leqslant c_{5} \frac{\left\|\widehat{u}_{n}+\bar{u}_{n}\right\|}{\left\|u_{n}\right\|^{\alpha}} \quad \forall n \geqslant 1,
$$

for some $c_{5}>0$. Using (3.18) in (3.17), we have

$$
\left|\int_{\Omega} \frac{f_{\infty}\left(z, u_{n}\right)\left(\widehat{u}_{n}+\bar{u}_{n}\right)}{\left\|u_{n}\right\|^{2 \alpha}} \mathrm{d} z\right| \leqslant c_{6} \frac{\left\|\widehat{u}_{n}+\bar{u}_{n}\right\|}{\left\|u_{n}\right\|^{\alpha}} \quad \forall n \geqslant n_{0},
$$


for some $n_{0} \geqslant 1$ and $c_{6}>0$, so

$$
\lim _{n \rightarrow+\infty} \int_{\Omega} \frac{f_{\infty}\left(z, u_{n}\right)\left(\widehat{u}_{n}+\bar{u}_{n}\right)}{\left\|u_{n}\right\|^{2 \alpha}} \mathrm{d} z=0
$$

(see (3.13)). Therefore

$$
\begin{aligned}
& \limsup _{n \rightarrow+\infty} \int_{\Omega} \frac{f_{\infty}\left(z, u_{n}\right) u_{n}^{0}}{\left\|u_{n}\right\|^{2 \alpha}} \mathrm{d} z \\
& \quad=\limsup _{n \rightarrow+\infty}\left(\int_{\Omega} \frac{f_{\infty}\left(z, u_{n}\right) u_{n}}{\left\|u_{n}\right\|^{2 \alpha}} \mathrm{d} z-\int_{\Omega} \frac{f_{\infty}\left(z, u_{n}\right)\left(\widehat{u}_{n}+\bar{u}_{n}\right)}{\left\|u_{n}\right\|^{2 \alpha}} \mathrm{d} z\right) \\
& \quad \leqslant \limsup _{n \rightarrow+\infty} \int_{\Omega} \frac{f_{\infty}\left(z, u_{n}\right) u_{n}}{\left\|u_{n}\right\|^{2 \alpha}} \mathrm{d} z<0
\end{aligned}
$$

(see (3.19) and Proposition 3.3). Comparing (3.15) and (3.26), we reach a contradiction. This proves the proposition.

Using this proposition, we can now establish the Cerami condition for the energy functional $\varphi$.

Proposition 3.5 If hypotheses $H_{f}(\mathrm{i})$ and (ii) hold, then $\varphi$ satisfies the Cerami condition.

Proof Let $\left\{u_{n}\right\}_{n \geqslant 1} \subseteq H_{n}^{1}(\Omega)$ be a sequence, such that $\left\{\varphi\left(u_{n}\right)\right\}_{n \geqslant 1} \subseteq \mathbb{R}$ is bounded and

$$
\left(1+\left\|u_{n}\right\|\right) \varphi^{\prime}\left(u_{n}\right) \longrightarrow 0 \text { in } H_{n}^{1}(\Omega)^{*} .
$$

We show that the sequence $\left\{u_{n}\right\}_{n} \geqslant 1 \subseteq H_{n}^{1}(\Omega)$ is bounded. We argue indirectly. So, suppose that by passing to a suitable subsequence if necessary, we have $\left\|u_{n}\right\| \rightarrow+\infty$. Note that (3.21) implies

$$
\left|\left\langle\varphi^{\prime}\left(u_{n}\right), h\right\rangle\right| \leqslant \frac{\varepsilon_{n}\|h\|}{1+\left\|u_{n}\right\|} \quad \forall h \in H_{n}^{1}(\Omega)
$$

with $\varepsilon_{n} \searrow 0$. In (3.22), we choose $h=\widehat{u}_{n} \in H_{n}^{1}(\Omega)$ and exploiting the orthogonality of the component spaces, we have

$$
\left\langle\varphi^{\prime}\left(u_{n}\right), \widehat{u}_{n}\right\rangle=\left\|\nabla \widehat{u}_{n}\right\|_{2}^{2}-\widehat{\lambda}_{i}\left\|\widehat{u}_{n}\right\|_{2}^{2}-\int_{\Omega} f_{\infty}\left(z, u_{n}\right) \widehat{u}_{n} \mathrm{~d} z \leqslant \varepsilon_{n},
$$

so

$$
\begin{aligned}
\xi_{0}\left\|\widehat{u}_{n}\right\|^{2} & \leqslant \varepsilon_{n}+\int_{\Omega} f_{\infty}\left(z, u_{n}\right) \widehat{u}_{n} \mathrm{~d} z \\
& \leqslant \varepsilon_{n}+\int_{\Omega}\left(\varepsilon\left|u_{n}\right|^{\alpha}+c_{3}\right)\left|\widehat{u}_{n}\right| \mathrm{d} z \quad \forall n \geqslant 1
\end{aligned}
$$

(see Proposition 2.5(a) and (3.16)). Since

$$
\left|u_{n}\right|^{\alpha} \in L^{\frac{2}{\alpha}}(\Omega) \text { and }\left(\frac{2}{\alpha}\right)^{\prime}=\frac{2}{2-\alpha} \leqslant 2^{*},
$$


then from (3.23) and Hölder inequality, we have

$$
\xi_{0}\left\|\widehat{u}_{n}\right\|^{2} \leqslant \varepsilon_{n}+c_{7}\left(\varepsilon\left\|u_{n}\right\|^{\alpha}+1\right)\left\|\widehat{u}_{n}\right\| \quad \forall n \geqslant 1,
$$

for some $c_{7}>0$, so

$$
\xi_{0}\left(\frac{\left\|\widehat{u}_{n}\right\|}{\left\|u_{n}\right\|^{\alpha}}\right)^{2} \leqslant \frac{\varepsilon_{n}}{\left\|u_{n}\right\|^{2 \alpha}}+c_{7}\left(\varepsilon \frac{\left\|\widehat{u}_{n}\right\|}{\left\|u_{n}\right\|^{\alpha}}+\frac{\left\|\widehat{u}_{n}\right\|}{\left\|u_{n}\right\|^{2 \alpha}}\right) \quad \forall n \geqslant 1 .
$$

We claim that the sequence $\left\{\mu_{n}=\frac{\left\|\widehat{u}_{n}\right\|}{\left\|u_{n}\right\|^{\alpha}}\right\}_{n \geqslant 1}$ is bounded. Indeed, if $\mu_{n} \rightarrow+\infty$ (at least for a subsequence), then dividing (3.24) with $\mu_{n}^{2}$, we obtain

$$
\xi_{0} \leqslant \varepsilon_{n}^{\prime}+\varepsilon \frac{c_{7}}{\mu_{n}}+c_{7} \frac{1}{\mu_{n}},
$$

with $\varepsilon_{n}^{\prime} \searrow 0$. Passing to the limit as $n \rightarrow+\infty$, we obtain $\xi_{0} \leqslant 0$, a contradiction. Hence, the sequence $\left\{\mu_{n}\right\}_{n \geqslant 1}$ is bounded and we may assume that $\mu_{n} \longrightarrow \mu \geqslant 0$. Passing to the limit as $n \rightarrow+\infty$ in (3.24), we obtain

$$
\xi_{0} \mu^{2} \leqslant \varepsilon c_{7} \mu
$$

so

$$
\xi_{0} \mu \leqslant \varepsilon c_{7}
$$

Since $\varepsilon>0$ was arbitrary, we let $\varepsilon \searrow 0$, to conclude that $\mu=0$. Therefore,

$$
\frac{\widehat{u}_{n}}{\left\|u_{n}\right\|^{\alpha}} \longrightarrow 0 \text { in } H_{n}^{1}(\Omega)
$$

Next in (3.22), we choose $h=-\bar{u}_{n} \in \bar{H}_{i-1}$. Then reasoning as above, we obtain

$$
-\left\langle\varphi^{\prime}\left(u_{n}\right), \bar{u}_{n}\right\rangle=-\left\|\nabla \bar{u}_{n}\right\|_{2}^{2}+\widehat{\lambda}_{i}\left\|\bar{u}_{n}\right\|_{2}^{2}+\int_{\Omega} f_{\infty}\left(z, u_{n}\right) \bar{u}_{n} \mathrm{~d} z \leqslant \varepsilon_{n},
$$

so using Proposition 2.5(b), we have

$$
\xi_{1}\left\|\bar{u}_{n}\right\|^{2} \leqslant \varepsilon_{n}+\int_{\Omega}\left|f_{\infty}\left(z, u_{n}\right)\right|\left|\bar{u}_{n}\right| \mathrm{d} z \quad \forall n \geqslant 1 .
$$

Using also (3.16) and (3.24), we have

$$
\xi\left(\frac{\left\|\bar{u}_{n}\right\|}{\left\|u_{n}\right\|^{\alpha}}\right)^{2} \leqslant \frac{\varepsilon_{n}}{\left\|u_{n}\right\|^{2 \alpha}}+c_{7}\left(\varepsilon \frac{\left\|\bar{u}_{n}\right\|}{\left\|u_{n}\right\|^{\alpha}}+\frac{\left\|\bar{u}_{n}\right\|}{\left\|u_{n}\right\|^{2 \alpha}}\right) \quad \forall n \geqslant 1
$$

and so

$$
\frac{\bar{u}_{n}}{\left\|u_{n}\right\|^{\alpha}} \longrightarrow 0 \text { in } H_{n}^{1}(\Omega)
$$

(as before). Let $R>0, \vartheta \in(0,1)$ and $\widehat{\delta}>0$ be as postulated in Proposition 3.4. Then from (3.25), (3.26) and since $\left\|u_{n}\right\| \longrightarrow+\infty$, we have that $u_{n} \in C_{\infty}(R, \vartheta, \alpha)$ for all $n \geqslant n_{0}$ and so

$$
\left\langle\varphi^{\prime}\left(u_{n}\right), u_{n}^{0}\right\rangle \geqslant \widehat{\delta} \forall n \geqslant n_{0}
$$


(see Proposition 3.4). If in (3.22), we choose $h=u_{n}^{0} \in E\left(\widehat{\lambda}_{i}\right)$, then

$$
\left\langle\varphi^{\prime}\left(u_{n}\right), u_{n}^{0}\right\rangle \leqslant \varepsilon_{n},
$$

with $\varepsilon_{n} \searrow 0$. Comparing (3.27) and (3.28), we reach a contradiction. This proves that the sequence $\left\{u_{n}\right\}_{n} \geqslant 1 \subseteq H_{n}^{1}(\Omega)$ is bounded. So, we may assume that

$$
\begin{array}{ll}
u_{n} \stackrel{w}{\longrightarrow} u \quad \text { in } H_{n}^{1}(\Omega) \\
u_{n} \longrightarrow u \text { in } L^{2}(\Omega) .
\end{array}
$$

In (3.22), we choose $h=u_{n}-u \in H_{n}^{1}(\Omega)$. We have

$$
\left|\left\langle A\left(u_{n}\right), u_{n}-u\right\rangle-\int_{\Omega} f\left(z, u_{n}\right)\left(u_{n}-u\right) \mathrm{d} z\right| \leqslant \frac{\varepsilon_{n}\left\|u_{n}-u\right\|}{1+\left\|u_{n}\right\|},
$$

so

$$
\lim _{r \rightarrow+\infty}\left\langle A\left(u_{n}\right), u_{n}-u\right\rangle=0
$$

(see (3.29)), so

$$
\left\|\nabla u_{n}\right\|_{2}^{2} \longrightarrow\|\nabla u\|_{2}^{2}
$$

(since $A\left(u_{n}\right) \stackrel{w}{\longrightarrow} A(u)$ in $H_{n}^{1}(\Omega)^{*}$; see (3.29)). From the Kadec-Klee property of Hilbert spaces, we have

$$
\nabla u_{n} \longrightarrow \nabla u \text { in } L^{2}\left(\Omega ; \mathbb{R}^{N}\right),
$$

so

$$
u_{n} \longrightarrow u \text { in } H_{n}^{1}(\Omega)
$$

(see (3.29)). Therefore, $\varphi$ satisfies the Cerami condition.

\section{Critical groups}

Using the eigenvalue $\widehat{\lambda}_{m}>0$ from hypothesis $H_{f}$ (iii), we can have the following orthogonal direct sum decomposition of $H_{n}^{1}(\Omega)$ :

$$
H_{n}^{1}(\Omega)=\bar{H}_{m-1} \oplus E\left(\widehat{\lambda}_{m}\right) \oplus \widehat{H}_{m+1},
$$

where

$$
\bar{H}_{m-1}=\bigoplus_{k=0}^{m-1} E\left(\widehat{\lambda}_{k}\right) \text { and } \widehat{H}_{m+1}=\bigoplus_{k \geqslant m+1} E\left(\widehat{\lambda}_{k}\right) .
$$

Then for every $u \in H_{n}^{1}(\Omega)$, we have

$$
u=\bar{u}+u^{0}+\widehat{u},
$$

with $\bar{u} \in \bar{H}_{m-1}, u^{0} \in E\left(\widehat{\lambda}_{m}\right), \widehat{u} \in \widehat{H}_{m+1}$ and the decomposition is unique. Using hypothesis $H_{f}($ iii $)$, we can have a result analogous to Proposition 3.3. 
Proposition 4.1 If $\left\{u_{n}\right\}_{n} \geqslant 1 \subseteq H_{n}^{1}(\Omega)$ is a sequence, such that

$$
\left\|u_{n}\right\| \longrightarrow 0 \text { and } \frac{\widehat{u}_{n}+\bar{u}_{n}}{\left\|u_{n}\right\|} \longrightarrow 0 \text { in } H_{n}^{1}(\Omega),
$$

then

$$
\limsup _{n \rightarrow+\infty} \int_{\Omega} \frac{f_{0}\left(z, u_{n}\right) u_{n}}{\left\|u_{n}\right\|^{2 \beta}} d z<0 .
$$

Proof It is clear from hypothesis $H_{f}$ (iii) that we can take $\beta>1$ small, such that $2^{*}>2 \beta$. As in the proof of Proposition 3.3, from Bartolo et al. [3], we know that for a given $\varepsilon>0$, we can find $m_{1}(\varepsilon)>0$ small enough and $m_{2}(\varepsilon)>0$ large enough, such that

$$
\left|\left\{z \in \Omega:\left|u^{0}(z)\right|<m_{1}\left\|u^{0}\right\|\right\}\right|_{N}<\varepsilon \quad \forall u^{0} \in E\left(\widehat{\lambda}_{m}\right)
$$

and

$$
\begin{aligned}
& \left|\left\{z \in \Omega:|\widehat{u}(z)+\bar{u}(z)|>m_{2}\|\widehat{u}+\bar{u}\|\right\}\right|_{N} \\
& <m_{1}^{\frac{2^{*}}{22^{*}-2 \beta} 2 \beta} \varepsilon \leqslant \varepsilon \quad \forall \widehat{u} \in \widehat{H}_{m+1}, \bar{u} \in \bar{H}_{m-1} .
\end{aligned}
$$

For every $n \geqslant 1$, we introduce the following sets:

$$
\begin{aligned}
& \Omega_{1 n}=\left\{z \in \Omega:\left|u_{n}^{0}(z)\right| \geqslant m_{1}\left\|u_{n}^{0}\right\|\right\}, \\
& \Omega_{2 n}=\left\{z \in \Omega:\left|\widehat{u}_{n}(z)+\bar{u}_{n}(z)\right| \leqslant m_{2}\left\|\widehat{u}_{n}+\bar{u}_{n}\right\|\right\} .
\end{aligned}
$$

From (4.1) and (4.2), we have

$$
\left|\Omega \backslash \Omega_{1 n}\right|_{N}<\varepsilon, \quad\left|\Omega \backslash \Omega_{2 n}\right|_{N}<\varepsilon
$$

and

$$
\left|\Omega_{1 n} \cap \Omega_{1 n}\right|_{N} \geqslant\left|\Omega_{1 n}\right|_{N}-\left|\Omega \backslash \Omega_{2 n}\right|_{N} \geqslant|\Omega|_{N}-2 \varepsilon .
$$

Choosing $\varepsilon \in\left(0, \frac{1}{2}|\Omega|_{N}\right)$, we see that $\left|\Omega_{1 n} \cap \Omega_{2 n}\right|>0$, hence $\Omega_{1 n} \cap \Omega_{2 n} \neq 0$. Let $z \in \Omega_{1 n} \cap \Omega_{2 n}$. Then

$$
\begin{aligned}
\frac{\left|u_{n}(z)\right|}{\left\|u_{n}\right\|} & =\frac{\left|u_{n}^{0}(z)+\widehat{u}_{n}(z)+\bar{u}_{n}(z)\right|}{\left\|u_{n}\right\|} \geqslant \frac{\left|u_{n}^{0}(z)\right|}{\left\|u_{n}\right\|}-\frac{\left|\widehat{u}_{n}(z)-\bar{u}_{n}(z)\right|}{\left\|u_{n}\right\|} \\
& \geqslant m_{1} \frac{\left\|u_{n}^{0}\right\|}{\left\|u_{n}\right\|}-m_{2} \frac{\left\|\widehat{u}_{n}+\bar{u}_{n}\right\|}{\left\|u_{n}\right\|} .
\end{aligned}
$$

Next, let $z \in \Omega_{2 n} \backslash \Omega_{1 n}$. Then

$$
\begin{aligned}
\frac{\left|u_{n}(z)\right|}{\left\|u_{n}\right\|} & =\frac{\left|u_{n}^{0}(z)+\widehat{u}_{n}(z)+\bar{u}_{n}(z)\right|}{\left\|u_{n}\right\|} \leqslant \frac{\left|u_{n}^{0}(z)\right|}{\left\|u_{n}\right\|}+\frac{\left|\widehat{u}_{n}(z)+\bar{u}_{n}(z)\right|}{\left\|u_{n}\right\|} \\
& <m_{1} \frac{\left\|u_{n}^{0}\right\|}{\left\|u_{n}\right\|}+m_{2} \frac{\left\|\widehat{u}_{n}+\bar{u}_{n}\right\|}{\left\|u_{n}\right\|} .
\end{aligned}
$$

Hypothesis $H_{f}($ iii) implies that we can find $\delta=\delta(\varepsilon)>0$, such that

$$
f_{0}(z, \zeta) \zeta \leqslant\left(\eta_{0}(z)+m_{1}^{2 \beta} \varepsilon\right)|\zeta|^{2 \beta} \text { for almost all } z \in \Omega \text {, all }|\zeta| \leqslant \delta \text {. }
$$

On the other hand, by virtue of hypothesis $H_{f}(i)$, we have

$$
f_{0}(z, \zeta) \zeta \leqslant c_{8}|\zeta|^{\mu} \text { for almost all } z \in \Omega \text {, all }|\zeta|>\delta
$$


and some $c_{8}>0, \mu>2 \beta$. Combining (4.7) and (4.8), we infer that

$$
f_{0}(z, \zeta) \zeta \leqslant\left(\eta_{0}(z)+m_{1}^{2 \beta} \varepsilon\right)|\zeta|^{2 \beta}+c_{9}|\zeta|^{\mu} \text { for almost all } z \in \Omega \text {, all } \zeta \in \mathbb{R},
$$

for some $c_{9}>0$. It is clear from hypothesis $H_{f}(\mathrm{iii})$ that we can take $\beta>1$ small enough, so that $2 \beta<2^{*}$. Then, we can choose $\mu \in\left(\max \{2 \beta, r\}, 2^{*}\right)$.

Using (4.9) and recalling that $\eta_{0} \leqslant 0$, we have

$$
\begin{aligned}
& \int_{\Omega_{1 n} \cap \Omega_{2 n}} \frac{f_{0}\left(z, u_{n}\right) u_{n}}{\left\|u_{n}\right\|^{2 \beta}} \mathrm{d} z \\
& \leqslant \int_{\Omega_{1 n} \cap \Omega_{2 n}}\left(\frac{\left(\eta_{0}(z)+\varepsilon\right)\left|u_{n}\right|^{2 \beta}}{\left\|u_{n}\right\|^{2 \beta}}+c_{9} \frac{\left|u_{n}\right|^{\mu}}{\left\|u_{n}\right\|^{2 \beta}}\right) \mathrm{d} z \\
& \leqslant\left(m_{1} \frac{\left\|u_{n}^{0}\right\|}{\left\|u_{n}\right\|}-m_{2} \frac{\left\|\widehat{u}_{n}+\bar{u}_{n}\right\|}{\left\|u_{n}\right\|}\right)^{2 \beta} \int_{\Omega_{1 n} \cap \Omega_{2 n}} \eta_{0}(z) \mathrm{d} z \\
& +\varepsilon \int_{\Omega_{1 n} \cap \Omega_{2 n}}\left(\frac{\left|u_{n}\right|}{\left\|u_{n}\right\|}\right)^{2 \beta} \mathrm{d} z \\
& +c_{9} \int_{\Omega_{1 n} \cap \Omega_{2 n}}\left(\frac{\left|u_{n}\right|}{\left\|u_{n}\right\|}\right)^{2 \beta}\left|u_{n}\right|^{\mu-2 \beta} \mathrm{d} z .
\end{aligned}
$$

Note that

$$
\left(\frac{\left|u_{n}\right|}{\left\|u_{n}\right\|}\right)^{2 \beta} \subseteq L^{\frac{\mu}{2 \beta}}(\Omega) \text { and }\left|u_{n}\right|^{\mu-2 \beta} \in L^{\frac{\mu}{\mu-2 \beta}}(\Omega) .
$$

Moreover, we have

$$
\frac{2 \beta}{\mu}+\frac{\mu-2 \beta}{\mu}=1 .
$$

So, applying Hölder inequality, we have

$$
\int_{\Omega_{1 n} \cap \Omega_{2 n}}\left(\frac{\left|u_{n}\right|}{\left\|u_{n}\right\|}\right)^{2 \beta}\left|u_{n}\right|^{\mu-2 \beta} \mathrm{d} z \leqslant c_{10}\left\|\chi_{\Omega_{1 n} \cap \Omega_{2 n}} u_{n}\right\|_{\mu}^{\mu-2 \beta} \quad \forall n \geqslant 1,
$$

for some $c_{10}>0$. In addition, we have

$$
\left(m_{1} \frac{\left\|u_{n}^{0}\right\|}{\left\|u_{n}\right\|}-m_{2} \frac{\left\|\widehat{u}_{n}+\bar{u}_{n}\right\|}{\left\|u_{n}\right\|}\right)^{2 \beta} \geqslant \frac{m_{1}^{2 \beta}}{4^{\beta}}\left(\frac{\left\|u_{n}^{0}\right\|}{\left\|u_{n}\right\|}\right)^{2 \beta}-m_{2}^{2 \beta} \frac{\left\|\widehat{u}_{n}+\bar{u}_{n}\right\|^{2 \beta}}{\left\|u_{n}\right\|^{2 \beta}} .
$$

We return to (4.10) and use (4.11), (4.12), to obtain

$$
\begin{aligned}
& \int_{\Omega_{1 n} \cap \Omega_{2 n}} \frac{f_{0}\left(z, u_{n}\right) u_{n}}{\left\|u_{n}\right\|^{2 \beta}} \mathrm{d} z \\
& \leqslant \frac{m_{1}^{2 \beta}}{4^{\beta}}\left(\frac{\left\|u_{n}^{0}\right\|}{\left\|u_{n}\right\|}\right)^{2 \beta} \int_{\Omega_{1 n} \cap \Omega_{2 n}} \eta_{0}(z) \mathrm{d} z+m_{2}^{2 \beta}\left(\frac{\left\|\widehat{u}_{n}+\bar{u}_{n}\right\|}{\left\|u_{n}\right\|}\right)^{2 \beta}\left\|\eta_{0}\right\|_{1} \\
& \quad+m_{1}^{2 \beta} \varepsilon c_{11}+c_{10}\left\|\chi_{\Omega_{1 n} \cap \Omega_{2 n}} u_{n}\right\|_{\mu}^{\mu-2 \beta} \quad \forall n \geq 1,
\end{aligned}
$$


for some $c_{11}>0$ (recall that $\int_{\Omega_{1 n} \cap \Omega_{2 n}} \eta_{0} \mathrm{~d} z \leqslant 0$ ). Hypotheses $H_{f}($ i $)$ and (iii) imply that

$$
f_{0}(z, \zeta) \zeta \leqslant c_{12}\left(|\zeta|^{2 \beta}+|\zeta|^{\mu}\right) \text { for almost all } z \in \Omega \text {, all } \zeta \in \mathbb{R}
$$

and some $c_{12}>0$. Hence, we have

$$
\begin{aligned}
& \int_{\Omega_{2 n} \backslash \Omega_{1 n}} \frac{f_{0}\left(z, u_{n}\right) u_{n}}{\left\|u_{n}\right\|^{2 \beta} \mathrm{d} z} \\
& \leqslant c_{12} \int_{\Omega_{2 n} \backslash \Omega_{1 n}}\left(\frac{\left|u_{n}\right|}{\left\|u_{n}\right\|}\right)^{2 \beta}\left(1+\left|u_{n}\right|^{\mu-2 \beta}\right) \mathrm{d} z \\
& \leqslant c_{12}\left(m_{1} \frac{\left\|u_{n}^{0}\right\|}{\left\|u_{n}\right\|}+m_{2} \frac{\left\|\widehat{u}_{n}+\bar{u}_{n}\right\|}{\left\|u_{n}\right\|}\right)^{2 \beta} \int_{\Omega_{2 n} \backslash \Omega_{1 n}}\left(1+\left|u_{n}\right|^{\mu-2 \beta}\right) \mathrm{d} z \\
& \leqslant 4^{\beta} c_{12}\left(m_{1}^{2 \beta}\left(\frac{\left\|u_{n}^{0}\right\|}{\left\|u_{n}\right\|}\right)^{2 \beta}+m_{2}^{2 \beta}\left(\frac{\| \widehat{u}_{n}+\bar{u}_{n}}{\left\|u_{n}\right\|}\right)^{2 \beta}\right)_{\Omega_{2 n} \backslash \Omega_{1 n}}\left(1+\left|u_{n}\right|^{\mu-2 \beta}\right) \mathrm{d} z \\
& \leqslant c_{13}\left(m_{1}^{2 \beta}\left(\frac{\left\|u_{n}^{0}\right\|}{\left\|u_{n}\right\|}\right)^{2 \beta}+m_{2}^{2 \beta}\left(\frac{\| \widehat{u}_{n}+\bar{u}_{n}}{\left\|u_{n}\right\|}\right)^{2 \beta}\right)_{\left|\Omega_{2 n} \backslash \Omega_{1 n}\right|_{N}^{\frac{2 \beta}{\mu}}} \\
& \leqslant \varepsilon^{\frac{2 \beta}{\mu}} c_{13}\left(m_{1}^{2 \beta}\left(\frac{\left\|u_{n}^{0}\right\|}{\left\|u_{n}\right\|}\right)^{2 \beta}+m_{2}^{2 \beta}\left(\frac{\left\|\widehat{u}_{n}+\bar{u}_{n}\right\|}{\left\|u_{n}\right\|}\right)^{2 \beta}\right) \forall n \geqslant 1 .
\end{aligned}
$$

for some $c_{13}>0$ (see (4.6) and (4.3)). Similarly, we have

$$
\begin{aligned}
& \int_{\Omega \backslash \Omega_{2 n}} \frac{f_{0}\left(z, u_{n}\right) u_{n}}{\left\|u_{n}\right\|} \mathrm{d} z \\
& \leqslant c_{12} \int_{\Omega \backslash \Omega_{2 n}}\left(\frac{\left|u_{n}\right|}{\left\|u_{n}\right\|}\right)^{2 \beta} \mathrm{d} z+c_{12} \int_{\Omega \backslash \Omega_{2 n}}\left(\frac{\left|u_{n}\right|}{\left\|u_{n}\right\|}\right)^{2 \beta}\left|u_{n}\right|^{\mu-2 \beta} \mathrm{d} z \\
& \leqslant c_{14}\left|\Omega \backslash \Omega_{2 n}\right|_{N}^{\frac{2^{*}-2 \beta}{2^{*}}} \leqslant c_{15} m_{1}^{2 \beta} \varepsilon,
\end{aligned}
$$

for some $c_{14}, c_{15}>0$ (see and (4.2) (4.14)). From (4.13), (4.15), and (4.16), we have

$$
\begin{aligned}
& \int_{\Omega} \frac{f_{0}\left(z, u_{n}\right) u_{n}}{\left\|u_{n}\right\|^{2 \beta}} \mathrm{d} z \\
& \leqslant \frac{m_{1}^{2 \beta}}{4^{\beta}}\left(\frac{\left\|u_{n}^{0}\right\|}{\left\|u_{n}\right\|}\right)^{2 \beta} \int_{\Omega_{1 n} \cap \Omega_{2 n}} \eta_{0}(z) \mathrm{d} z+m_{2}^{2 \beta}\left(\frac{\left\|\widehat{u}_{n}+\bar{u}_{n}\right\|}{\left\|u_{n}\right\|}\right)^{2 \beta}\left\|\eta_{0}\right\|_{1} \\
& \quad+c_{10}\left\|\chi_{\Omega_{1 n} \cap \Omega_{2 n}} u_{n}\right\|_{\mu}^{\mu-2 \beta} \\
& \quad+\varepsilon^{\frac{2 \beta}{\mu}} c_{13}\left(m_{1}^{2 \beta}\left(\frac{\left\|u_{n}^{0}\right\|}{\left\|u_{n}\right\|}\right)^{2 \beta}+m_{2}^{2 \beta}\left(\frac{\left\|\widehat{u}_{n}+\bar{u}_{n}\right\|}{\left\|u_{n}\right\|}\right)^{2 \beta}\right) \\
& \quad+m_{1}^{2 \beta} \varepsilon\left(c_{11}+c_{15}\right) .
\end{aligned}
$$

Note that for all $n \geqslant 1$, we have

$$
\chi_{\Omega_{1 n} \cap \Omega_{2 n}}(z) \longrightarrow \chi_{\Omega}(z)=1 \text { almost everywhere on } \Omega \text { as } \varepsilon \searrow 0
$$


(see (4.3)). So, if in (4.17) we pass to the limit as $n \rightarrow+\infty$, then

$$
\limsup _{n \rightarrow+\infty} \int_{\Omega} \frac{f_{0}\left(z, u_{n}\right) u_{n}}{\left\|u_{n}\right\|^{2 \beta}} \mathrm{d} z \leqslant m_{1}^{2 \beta}\left(\frac{1}{4^{\beta}} \int_{\Omega} \eta_{0}(z) \mathrm{d} z+\varepsilon\left(c_{11}+c_{15}+c_{13}\right)\right)
$$

Choosing $\varepsilon \in(0,1)$ small enough, we have

$$
\limsup _{n \rightarrow+\infty} \int_{\Omega} \frac{f_{0}\left(z, u_{n}\right) u_{n}}{\left\|u_{n}\right\|^{2 \beta}} \mathrm{d} z<0 .
$$

This proposition leads to the introduction of a certain set similar to the set $C_{\infty}(R, \vartheta, \alpha)$. So, for $\varrho>0$ and $\vartheta \in(0,1)$, we introduce the set $C_{0}(\varrho, \vartheta, \beta)$, defined by

$$
C_{0}(\varrho, \vartheta, \beta)=\left\{u \in H_{n}^{1}(\Omega):\|u\| \leqslant \varrho,\|\widehat{u}+\bar{u}\| \leqslant \vartheta\|u\|^{\beta}\right\} .
$$

For this set, we prove a result analogous to Proposition 3.4.

Proposition 4.2 If hypotheses $H_{f}$ (i) and (iii) hold, then there exist $\varrho>0$ and $\vartheta \in(0,1)$ such that

$$
\left\langle\varphi^{\prime}(u), u^{0}\right\rangle \geqslant 0 \quad \forall u \in C_{0}(\varrho, \vartheta, \beta) .
$$

Proof We proceed by contradiction. So, suppose that for every $\varrho=\vartheta=\frac{1}{n}, n \geqslant 1$, we can find $u_{n} \in H_{n}^{1}(\Omega)$, such that

$$
\left\|u_{n}\right\| \leqslant \frac{1}{n}, \quad\left\|\widehat{u}_{n}+\bar{u}_{n}\right\| \leqslant \frac{1}{n}\left\|u_{n}\right\|^{\beta} \quad \text { and } \quad\left\langle\varphi^{\prime}\left(u_{n}\right), u_{n}^{0}\right\rangle<0 \quad \forall n \geqslant 1
$$

From (4.18), we have

$$
\left\|u_{n}\right\| \longrightarrow 0 \text { and } \frac{\left\|\widehat{u}_{n}+\bar{u}_{n}\right\|}{\left\|u_{n}\right\|^{\beta}} \longrightarrow 0 .
$$

From the last inequality in (4.18), we have

$$
\begin{aligned}
\left\langle\varphi^{\prime}\left(u_{n}\right), u_{n}^{0}\right\rangle & =\left\langle A\left(u_{n}\right), u_{n}^{0}\right\rangle-\int_{\Omega}\left(\widehat{\lambda}_{m} u_{n}+f\left(z, u_{n}\right)\right) u_{n}^{0} \mathrm{~d} z \\
& =\left\|\nabla u_{n}^{0}\right\|_{2}^{2}-\widehat{\lambda}_{m}\left\|u_{n}^{0}\right\|_{2}^{2}-\int_{\Omega} f_{0}\left(z, u_{n}\right) u_{n}^{0} \mathrm{~d} z \\
& =-\int_{\Omega} f_{0}\left(z, u_{n}\right) u_{n}^{0} \mathrm{~d} z<0 \quad \forall n \geqslant 1
\end{aligned}
$$

(recall that $u_{n}^{0} \in E\left(\widehat{\lambda}_{m}\right)$ ), so

$$
\liminf _{n \rightarrow+\infty} \int_{\Omega} \frac{f_{0}\left(z, u_{n}\right) u_{n}^{0}}{\left\|u_{n}\right\|^{2 \beta}} \mathrm{d} z \geqslant 0 .
$$

Hypotheses $H_{f}(i)$ and (iii) imply that we can find $c_{16}=c_{16}(\varepsilon)>0$, such that

$$
\left|f_{0}(z, \zeta)\right| \leqslant \varepsilon|\zeta|^{\beta}+c_{16}|\zeta|^{\tau} \text { for almost all } z \in \Omega \text {, all } \zeta \in \mathbb{R},
$$

with $\tau \in\left(1,2^{*}-1\right)$. As before (see the proof of Proposition 4.1), we may assume that $\beta>1$ is small enough, so that $2 \beta<2^{*}$ and $\beta \leqslant \tau$. Then 


$$
\begin{aligned}
& \left|\int_{\Omega} \frac{f_{0}\left(z, u_{n}\right)\left(\widehat{u}_{n}+\bar{u}_{n}\right)}{\left\|u_{n}\right\|^{2 \beta}} \mathrm{d} z\right| \\
& \quad \leqslant \int_{\Omega} \frac{\varepsilon\left|u_{n}\right|^{\beta}\left|\widehat{u}_{n}+\bar{u}_{n}\right|}{\left\|u_{n}\right\|^{2 \beta}} \mathrm{d} z+c_{16} \int_{\Omega} \frac{\left|u_{n}\right|^{\tau}\left|\widehat{u}_{n}+\bar{u}_{n}\right|}{\left\|u_{n}\right\|^{2 \beta}} \mathrm{d} z
\end{aligned}
$$

(see (4.21)). Note that

$$
\left|u_{n}\right|^{\beta} \in L^{\frac{2^{*}}{\beta}}(\Omega) \text { and }\left(\frac{2^{*}}{\beta}\right)^{\prime}=\frac{2^{*}}{2^{*}-\beta}<2^{*},
$$

hence $\left|\widehat{u}_{n}+\bar{u}\right| \in L^{\frac{2^{*}}{2^{*}-\beta}}(\Omega)$. So, using Hölder inequality, we have

$$
\begin{aligned}
\int_{\Omega}\left|u_{n}\right|^{\beta}\left|\widehat{u}_{n}+\bar{u}_{n}\right| \mathrm{d} z & \leqslant\left(\int_{\Omega}\left|u_{n}\right|^{2^{*}} \mathrm{~d} z\right)^{\frac{\beta}{2^{*}}}\left(\int_{\Omega}\left|\widehat{u}_{n}+\bar{u}_{n}\right|^{\frac{2^{*}}{2^{*}-\beta}} \mathrm{d} z\right)^{\frac{2^{*}-\beta}{2^{*}}} \\
& \leqslant c_{17}\left\|u_{n}\right\|^{\beta}\left\|\widehat{u}_{n}+\bar{u}_{n}\right\| \quad \forall n \geqslant 1,
\end{aligned}
$$

for some $c_{17}>0$. Also

$$
\left|u_{n}\right|^{\tau} \in L^{\frac{2^{*}}{\tau}}(\Omega) \text { and }\left(\frac{2^{*}}{\tau}\right)^{\prime}=\frac{2^{*}}{2^{*}-\tau}<2^{*}
$$

(since $\left.\tau=r-1<2^{*}-1\right)$. Hence,

$$
\int_{\Omega}\left|u_{n}\right|^{\tau}\left|\widehat{u}_{n}+\bar{u}_{n}\right| \mathrm{d} z \leqslant c_{18}\left\|u_{n}\right\|^{\tau}\left\|\widehat{u}_{n}+\bar{u}_{n}\right\| \quad \forall n \geqslant 1,
$$

for some $c_{18}>0$. Returning to (4.22) and using (4.23) and (4.24), we obtain

$$
\begin{aligned}
& \left|\int_{\Omega} \frac{f_{0}\left(z, u_{n}\right)\left(\widehat{u}_{n}+\bar{u}_{n}\right)}{\left\|u_{n}\right\|^{2 \beta}} \mathrm{d} z\right| \\
& \quad \leqslant \varepsilon c_{17} \frac{\left\|\widehat{u}_{n}+\bar{u}_{n}\right\|}{\left\|u_{n}\right\|^{\beta}}+c_{18}\left\|u_{n}\right\|^{\tau-\beta} \frac{\left\|\widehat{u}_{n}+\bar{u}_{n}\right\|}{\left\|u_{n}\right\|^{\beta}} \quad \forall n \geqslant 1 .
\end{aligned}
$$

Passing to the limit as $n \rightarrow+\infty$ in (4.25) and recalling that $\tau \geqslant \beta$ and that $\varepsilon>0$ is arbitrary, we conclude that

$$
\lim _{n \rightarrow+\infty} \int_{\Omega} \frac{f_{0}\left(z, u_{n}\right)\left(\widehat{u}_{n}+\bar{u}_{n}\right)}{\left\|u_{n}\right\|^{2 \beta}} \mathrm{d} z=0 .
$$

Therefore, finally we have

$$
\begin{aligned}
& \liminf _{n \rightarrow+\infty} \int_{\Omega} \frac{f_{0}\left(z, u_{n}\right) u_{n}}{\left\|u_{n}\right\|^{2 \beta}} \mathrm{d} z \\
& \quad=\liminf _{n \rightarrow+\infty}\left(\int_{\Omega} \frac{f_{0}\left(z, u_{n}\right) u_{n}^{0}}{\left\|u_{n}\right\|^{2 \beta}} \mathrm{d} z+\int_{\Omega} \frac{f_{0}\left(z, u_{n}\right)\left(\widehat{u}_{n}+\bar{u}_{n}\right)}{\left\|u_{n}\right\|^{2 \beta}} \mathrm{d} z\right) \geqslant 0
\end{aligned}
$$

(see (4.20)) and (4.26). But this contradicts Proposition 4.1. 
Using this proposition, we can compute the critical groups of $\varphi$ at the origin.

Proposition 4.3 If hypotheses $H_{f}(\mathrm{i})$ and (iii) hold, then

$$
C_{k}(\varphi, 0)=\delta_{k, d_{m}} \mathbb{Z} \quad \forall k \geqslant 0 \text { and } d_{m}=\operatorname{dim} \bar{H}_{m-1} .
$$

Proof Let $\lambda \in\left(0, \widehat{\lambda}_{m}-\widehat{\lambda}_{m-1}\right)$ (recall that $\left.m \geqslant 1\right)$ and consider the homotopy

$$
h_{t}(u)=\varphi(u)+t \frac{\lambda}{2}\left\|u^{0}\right\|^{2} \quad \forall t \in[0,1] .
$$

Claim 1 There exists $\varrho_{0}>0$ small enough, such that $u=0$ is the only critical point of $h_{t}$ in $\bar{B}_{\varrho_{0}}=\left\{u \in H_{n}^{1}(\Omega):\|u\| \leqslant \varrho_{0}\right\}$ for all $t \in[0,1]$.

Suppose that the Claim is not true. Then, we can find two sequences $\left\{t_{n}\right\}_{n} \geqslant 1 \subseteq[0,1]$ and $\left\{u_{n}\right\}_{n \geqslant 1} \subseteq H_{n}^{1}(\Omega)$, such that

$$
t_{n} \longrightarrow t \in[0,1], \quad\left\|u_{n}\right\| \longrightarrow 0 \text { and }\left(h_{t_{n}}\right)^{\prime}\left(u_{n}\right)=0 \quad \forall n \geqslant 1 .
$$

From the equation in (4.27), we have

$$
\varphi^{\prime}\left(u_{n}\right)=-t_{n} \lambda u_{n}^{0},
$$

so

$$
\left\langle\varphi^{\prime}\left(u_{n}\right), u_{n}^{0}\right\rangle=-t_{n} \lambda\left\|u_{n}^{0}\right\|_{2}^{2} .
$$

Suppose that $u_{n}^{0}=0$ for all $n \geqslant n_{0}$. Then from (4.28), we have

$$
\varphi^{\prime}\left(u_{n}\right)=0 \quad \forall n \geqslant n_{0},
$$

so

$$
A\left(u_{n}\right)=N_{f}\left(u_{n}\right) \quad \forall n \geqslant n_{0} .
$$

Let $y_{n}=\frac{u_{n}}{\left\|u_{n}\right\|}$ for $n \geqslant 1$. Then $\left\|y_{n}\right\|=1$ for all $n \geqslant 1$ and so we may assume that

$$
\begin{array}{ll}
y_{n} \stackrel{w}{\longrightarrow} y & \text { in } H_{n}^{1}(\Omega), \\
y_{n} \longrightarrow y & \text { in } L^{2}(\Omega) .
\end{array}
$$

From (4.30), we have

$$
A\left(y_{n}\right)=\frac{N_{f}\left(u_{n}\right)}{\left\|u_{n}\right\|} \quad \forall n \geqslant n_{0} .
$$

Evidently the sequence $\left\{\frac{N_{f}\left(u_{n}\right)}{\left\|u_{n}\right\|}\right\} \subseteq L^{r^{\prime}}(\Omega)$ is bounded and so we may assume that

$$
\frac{N_{f}\left(u_{n}\right)}{\left\|u_{n}\right\|} \stackrel{w}{\longrightarrow} h \text { in } L^{r^{\prime}}(\Omega) .
$$

Moreover, using hypothesis $H_{f}(i i i)$, as in Aizicovici et al. [1] (see the proof of Proposition 31), we can show that

$$
h=\widehat{\lambda}_{m} y .
$$

So, if in (4.33) we pass to the limit as $n \rightarrow+\infty$, then from (4.31), (4.34), (4.35), we have

$$
A(y)=\widehat{\lambda}_{m} y,
$$


So

$$
\left\{\begin{array}{l}
-\Delta u(z)=\widehat{\lambda}_{m} y(z) \text { in } \Omega, \\
\frac{\partial u}{\partial n}=0 \text { on } \partial \Omega
\end{array}\right.
$$

and thus $y \in E\left(\widehat{\lambda}_{m}\right)$. Also, if on (4.33) we act with $y_{n}-y \in H_{n}^{1}(\Omega)$ and pass to the limit as $n \rightarrow+\infty$, then

$$
\lim _{n \rightarrow+\infty}\left\langle A\left(y_{n}\right), y_{n}-y\right\rangle=0,
$$

so

$$
y_{n} \longrightarrow y \text { in } H_{n}^{1}(\Omega)
$$

(see the end of the proof of Proposition 3.5). Thus, $y \in E\left(\widehat{\lambda}_{m}\right) \backslash\{0\}$ since $\|y\|=1$ and so $y=y^{0} \neq 0$.

Then

$$
\frac{u_{n}^{0}}{\left\|u_{n}^{0}\right\|}=y_{n}^{0} \longrightarrow y^{0}=y \neq 0 \text { in } H_{n}^{1}(\Omega),
$$

contradicting our standing hypothesis that $u_{n}^{0}=0$ for all $n \geqslant n_{0}$. So, by passing to a suitable subsequence if necessary, we may assume that $u_{n}^{0} \neq 0$ for all $n \geqslant 1$.

Let $\varrho>0$ and $\vartheta \in(0,1)$ be as postulated by Proposition 4.2 and suppose that $u_{n} \in C_{0}(\varrho, \vartheta, \beta)$ for all $n \geqslant \widehat{n}_{0} \geqslant 1$. Then by virtue of Proposition 4.2, we have

$$
\left\langle\varphi^{\prime}\left(u_{n}\right), u_{n}^{0}\right\rangle \geqslant 0 \quad \forall n \geqslant \widehat{n}_{0},
$$

which contradicts (4.29) (recall that $u_{n}^{0} \neq 0$ for all $n \geqslant 1$ ). So, at least for a subsequence, we have $u_{n} \notin C_{0}(\varrho, \vartheta, \beta)$ for all $n \geqslant 1$, hence

$$
\left\|\widehat{u}_{n}+\bar{u}_{n}\right\|>\vartheta\left\|u_{n}\right\|^{\beta} \quad \forall n \geqslant 1 .
$$

Exploiting the orthogonality of the component spaces and (4.27), we have

$$
\begin{aligned}
0= & \left\langle\left(h_{t_{n}}\right)^{\prime}\left(u_{n}\right), \widehat{u}_{n}-\bar{u}_{n}\right\rangle=\left\langle\varphi^{\prime}\left(u_{n}\right), \widehat{u}_{n}-\bar{u}_{n}\right\rangle \\
= & \left\|\nabla \widehat{u}_{n}\right\|_{2}^{2}-\widehat{\lambda}_{m}\left\|\widehat{u}_{n}\right\|_{2}^{2}-\left\|\nabla \bar{u}_{n}\right\|_{2}^{2}+\widehat{\lambda}_{m}\left\|\bar{u}_{n}\right\|_{2}^{2} \\
& -\int_{\Omega} f_{0}\left(z, u_{n}\right)\left|\widehat{u}_{n}-\bar{u}_{n}\right| \mathrm{d} z .
\end{aligned}
$$

As in the proof of Proposition 4.2, using (4.14), we obtain

$$
\begin{aligned}
& -\int_{\Omega} f_{0}\left(z, u_{n}\right)\left(\widehat{u}_{n}-\bar{u}_{n}\right) \mathrm{d} z \\
& \quad \geqslant-\varepsilon c_{19}\left\|u_{n}\right\|^{\beta}\left\|\widehat{u}_{n}+\bar{u}_{n}\right\|-c_{19}\left\|u_{n}\right\|^{\tau}\left\|\widehat{u}_{n}+\bar{u}_{n}\right\| \quad \forall n \geqslant 1,
\end{aligned}
$$

for some $c_{19}>0$ and with $\tau \in\left[\beta, 2^{*}-1\right.$ ) (see the proof of Proposition 4.2) and in particular (4.23) and (4.24) and note that by virtue of the orthogonality of the component spaces, we have $\left\|\widehat{u}_{n}-\bar{u}_{n}\right\|=\left\|\widehat{u}_{n}+\bar{u}_{n}\right\|$ for all $n \geqslant 1$. Using (4.38) in (4.37), we obtain

$$
\begin{aligned}
0 & \geqslant c_{20}\left\|\widehat{u}_{n}+\bar{u}_{n}\right\|^{2}-\varepsilon c_{19}\left\|u_{n}\right\|^{\beta}\left\|\widehat{u}_{n}+\bar{u}_{n}\right\|-c_{19}\left\|u_{n}\right\|^{\tau}\left\|\widehat{u}_{n}+\bar{u}_{n}\right\| \\
& =\left\|\widehat{u}_{n}+\bar{u}_{n}\right\|^{2}\left(c_{20}-\varepsilon c_{19} \frac{\left\|u_{n}\right\|^{\beta}}{\left\|\widehat{u}_{n}+\bar{u}_{n}\right\|}-c_{19}\left\|u_{n}\right\|^{\tau-\beta} \frac{\left\|u_{n}\right\|^{\beta}}{\left\|\widehat{u}_{n}+\bar{u}_{n}\right\|}\right) \\
& \geqslant\left\|\widehat{u}_{n}+\bar{u}_{n}\right\|^{2}\left(c_{20}-\varepsilon c_{19} \frac{1}{\vartheta}-c_{19}\left\|u_{n}\right\|^{\tau-\beta} \frac{1}{\vartheta}\right) \quad \forall n \geqslant 1
\end{aligned}
$$


for some $c_{20}>0$ (see Proposition 2.5 and (4.36)). Recall that $\left\|u_{n}\right\| \longrightarrow 0$ (see (4.27)). So, for $n \geqslant n_{0}$, we will have $\left\|u_{n}\right\|^{\tau-\beta}<\varepsilon$. Choosing $\varepsilon>0$ small enough, we have $c_{20}>2 \varepsilon c_{19} \frac{1}{\vartheta}$, which in conjunction with (4.39) leads to a contradiction. This proves the Claim.

Since

$$
\left\langle\left(h_{t}\right)^{\prime}(u), \widehat{u}\right\rangle=\left\langle\varphi^{\prime}(u), \widehat{u}\right\rangle \quad \forall u \in H_{n}^{1}(\Omega), t \in[0,1]
$$

and

$$
\left\langle\left(h_{t}\right)^{\prime}(u), \bar{u}\right\rangle=\left\langle\varphi^{\prime}(u), \bar{u}\right\rangle \quad \forall u \in H_{n}^{1}(\Omega), t \in[0,1]
$$

(a consequence of the orthogonality of the component spaces), as in the proof of Proposition 3.5, we check that for all $t \in[0,1], h_{t}(\cdot)$ satisfies the Cerami condition. This fact together with the Claim permits the use of the homotopy invariance property of critical groups (see e.g., [7, p. 332]). So, we have

$$
C_{k}\left(h_{0}, 0\right)=C_{k}\left(h_{1}, 0\right) \quad \forall k \geqslant 0,
$$

so

$$
C_{k}(\varphi, 0)=C_{k}\left(h_{1}, 0\right) \quad \forall k \geqslant 0 .
$$

Note that

$$
h_{1}(u)=\varphi(u)+\frac{\lambda}{2}\left\|u^{0}\right\|^{2} \quad \forall u \in H_{n}^{1}(\Omega)
$$

Evidently, $u=0$ is a critical point of $h_{1} \in C^{2}\left(H_{n}^{1}(\Omega)\right)$. We claim that $u=0$ is a nondegenerate critical point of $h_{1}$. Indeed, let $y \in \operatorname{ker} h_{1}^{\prime \prime}(0)$. Then

$$
h_{1}^{\prime \prime}(0) y=0 \text {, }
$$

so

$$
\varphi^{\prime \prime}(0) y+\lambda y=0
$$

and thus

$$
A(y)-\widehat{\lambda}_{m} y-\left(f_{0}\right)_{\zeta}^{\prime}(\cdot, 0) y+\lambda y=0 .
$$

Note that $f_{0}(z, 0)=0$ for almost all $z \in \Omega$ and

$$
\frac{f_{0}(z, \zeta)}{\zeta}=\frac{f(z, \zeta)}{\zeta}-\widehat{\lambda}_{m}
$$

So

$$
\lim _{\zeta \rightarrow 0} \frac{f_{0}(z, \zeta)}{\zeta}=\left(f_{0}\right)_{\zeta}^{\prime}(z, 0)=0 \text { uniformly for almost all } z \in \Omega
$$

(see hypothesis $H_{f}(i i i)$ ). Hence (4.41) becomes

$$
A(y)=\left(\widehat{\lambda}_{m}-\lambda\right) y,
$$

so

$$
\left\{\begin{array}{l}
-\Delta y(z)=\left(\widehat{\lambda}_{m}-\lambda\right) y(z) \text { in } \Omega \\
\frac{\partial y}{\partial n}=0 \text { on } \partial \Omega .
\end{array}\right.
$$


Note that

$$
\widehat{\lambda}_{m-1}<\widehat{\lambda}_{m}-\lambda<\widehat{\lambda}_{m},
$$

so

$$
\widehat{\lambda}_{m-1}\left(\widehat{\lambda}_{m}-\lambda\right)<\widehat{\lambda}_{m-1}\left(\widehat{\lambda}_{m-1}\right)=1 \text { and } \widehat{\lambda}_{m}\left(\widehat{\lambda}_{m}\right)=1<\widehat{\lambda}_{m}\left(\widehat{\lambda}_{m}-\lambda\right)
$$

(see Proposition 2.4). Then from (4.42), it follows that $y=0$ and so ker $h_{1}^{\prime \prime}(0)=\{0\}$, which implies that $u=0$ is a nondegenerate critical point of $h_{1}$. Also the Morse index of $u=0$ is $d_{m}=\operatorname{dim} \bar{H}_{m-1}$ (see Proposition 2.5). Therefore, it follows that

$$
C_{k}\left(h_{1}, 0\right)=\delta_{k, d_{m}} \mathbb{Z} \quad \forall k \geqslant 0,
$$

so

$$
C_{k}(\varphi, 0)=\delta_{k, d_{m}} \mathbb{Z} \quad \forall k \geqslant 0
$$

(see (4.40)).

Next using Proposition 2.6, we will compute the critical groups of $\varphi$ at infinity.

Proposition 4.4 If hypotheses $H_{f}$ (i) and (ii) hold, then

$$
C_{k}(\varphi, \infty)=\delta_{k, d_{l}} \mathbb{Z} \quad \forall k \geqslant 0,
$$

where $d_{i}=\operatorname{dim} \bar{H}_{i-1}$.

Proof We consider the homotopy $h_{t}$, defined by

$$
h_{t}(u)=\varphi(u)+\frac{t}{2}\left\|u^{0}\right\|^{2} \quad \forall u \in H_{n}^{1}(\Omega), t \in[0,1] .
$$

Evidently $h_{t} \in C^{2}\left(H_{n}^{1}(\Omega)\right)$ and $\left(h_{t}\right)^{\prime}$ and $\partial_{t} h_{t}$ are both locally Lipschitz for all $t \in[0,1]$.

Claim 2 There exists $a \in \mathbb{R}$ and $\delta>0$, such that

$$
h_{t}(u) \leqslant a \Longrightarrow(1+\|u\|)\left\|\left(h_{t}\right)^{\prime}(u)\right\|_{*} \geqslant \delta \quad \forall t \in[0,1] .
$$

Suppose that the Claim is not true. Since $h$ maps bounded sets to bounded ones, we can find two sequences $\left\{t_{n}\right\} \subseteq[0,1]$ and $\left\{u_{n}\right\} \subseteq H_{n}^{1}(\Omega)$, such that

$$
t_{n} \longrightarrow t \in[0,1], \quad\left\|u_{n}\right\| \longrightarrow+\infty, \quad, \quad h_{t_{n}}\left(u_{n}\right) \longrightarrow-\infty
$$

and

$$
\left(1+\left\|u_{n}\right\|\right)\left\|\left(h_{t_{n}}\right)^{\prime}\left(u_{n}\right)\right\|_{*} \longrightarrow 0 \text { in } H_{n}^{1}(\Omega)^{*}
$$

From (4.44), we have

$$
\left|\left\langle\left(h_{t_{n}}\right)^{\prime}\left(u_{n}\right), v\right\rangle\right| \leqslant \frac{\varepsilon_{n}}{1+\left\|u_{n}\right\|}\|v\| \quad \forall v \in H_{n}^{1}(\Omega),
$$

with $\varepsilon_{n} \searrow 0$, so

$$
\left|\left\langle A\left(u_{n}\right), v\right\rangle-\int_{\Omega} f\left(z, u_{n}\right) v \mathrm{~d} z+t_{n} \int_{\Omega} u_{n}^{0} v \mathrm{~d} z\right| \leqslant \frac{\varepsilon_{n}}{1+\left\|u_{n}\right\|}\|v\| \quad \forall n \geqslant 1 .
$$


Let

$$
y_{n}=\frac{u_{n}}{\left\|u_{n}\right\|} \quad \forall n \geqslant 1
$$

Then $\left\|y_{n}\right\|=1$ for all $n \geqslant 1$ and so we may assume that

$$
\begin{array}{ll}
y_{n} \stackrel{w}{\longrightarrow} y \text { in } H_{n}^{1}(\Omega) \\
y_{n} \longrightarrow y \text { in } L^{2}(\Omega) .
\end{array}
$$

From (4.45), we have

$$
\left|\left\langle A\left(y_{n}\right), v\right\rangle-\int_{\Omega} \frac{f\left(z, u_{n}\right)}{\left\|u_{n}\right\|} v \mathrm{~d} z+t_{n} \int_{\Omega} y_{n}^{0} v \mathrm{~d} z\right| \leqslant \frac{\varepsilon_{n}}{1+\left\|u_{n}\right\|}\|v\| \quad \forall n \geqslant 1 .
$$

By virtue of hypothesis $H_{f}(i i)$, we have

$$
\frac{f\left(\cdot, u_{n}(\cdot)\right)}{\left\|u_{n}\right\|} \stackrel{w}{\longrightarrow} \widehat{\lambda}_{i} y \text { in } L^{2}(\Omega)
$$

(see e.g., the proof of Proposition 22 of [1]). So, if in (4.48) we pass to the limit as $n \rightarrow+\infty$ and due (4.46) and (4.49), we obtain

$$
\langle A(y), v\rangle=\widehat{\lambda}_{i} \int_{\Omega} y v \mathrm{~d} z-t \int_{\Omega} y^{0} v \mathrm{~d} z \quad \forall v \in H_{n}^{1}(\Omega) .
$$

If in (4.48) we choose $v=y_{n}-y \in H_{n}^{1}(\Omega)$, pass to the limit as $n \rightarrow+\infty$ and use (4.46) and (4.49), we have

$$
\lim _{n \rightarrow+\infty}\left\langle A\left(y_{n}\right), y_{n}-y\right\rangle=0,
$$

so

$$
y_{n} \longrightarrow y \text { in } H_{n}^{1}(\Omega)
$$

(see the proof of Proposition 3.5), hence

$$
\|y\|=1 .
$$

In (4.50) we choose $v=\widehat{y} \in \widehat{H}_{i+1}$. Then

$$
\|\nabla \widehat{y}\|_{2}^{2}=\widehat{\lambda}_{i} \| \widehat{y}_{2}^{2},
$$

so

$$
\widehat{y}=0
$$

(since $\widehat{y} \in \widehat{H}_{i+1}$ ). Similarly, if in (4.50) we choose $v=\bar{y} \in \bar{H}_{i-1}$, then

$$
\|\nabla \bar{y}\|_{2}^{2}=\widehat{\lambda}_{i}\|\bar{y}\|_{2}^{2},
$$

so

$$
\bar{y}=0
$$

(since $\left.\bar{y} \in \bar{H}_{i-1}\right)$. From (4.51), (4.52) and (4.53), it follows that $y=y^{0} \in E\left(\widehat{\lambda}_{i}\right) \backslash\{0\}$. From (4.50), we have

$$
A(y)=\left(\widehat{\lambda}_{i}-t\right) y,
$$


So

$$
\|\nabla y\|_{2}^{2}=\widehat{\lambda}_{i}\|y\|_{2}^{2}-t\|y\|_{2}^{2}
$$

SO

$$
t\|y\|_{2}^{2}=0
$$

(since $\left.y=y^{0} \in E\left(\widehat{\lambda}_{i}\right) \backslash\{0\}\right)$ and thus $t=0$.

Reasoning as in the proof of Proposition 3.5, we show that

$$
\frac{\widehat{u}_{n}}{\left\|u_{n}\right\|^{\alpha}} \longrightarrow 0 \text { and } \frac{\bar{u}_{n}}{\left\|u_{n}\right\|^{\alpha}} \longrightarrow 0 \text { in } H_{n}^{1}(\Omega)
$$

(see (3.25) and (3.26)), so

$$
\frac{\widehat{u}_{n}+\bar{u}_{n}}{\left\|u_{n}\right\|^{\alpha}} \longrightarrow 0 \text { in } H_{n}^{1}(\Omega) .
$$

Hence, if $R>0, \vartheta \in(0,1]$ and $\widehat{\delta}>0$ are as postulated in Proposition 3.4, then

$$
u_{n} \in C_{\infty}(R, \vartheta, \alpha) \quad \forall n \geqslant n_{0}
$$

(see (4.37)), so

$$
\left\langle\varphi^{\prime}\left(u_{n}\right), u_{n}^{0}\right\rangle \geqslant \widehat{\delta}>0 \quad \forall n \geqslant n_{0}
$$

(see Proposition 2.5) and so

$$
\left\langle\left(h_{t_{n}}\right)^{\prime}\left(u_{n}\right), u_{n}^{0}\right\rangle \geqslant \frac{\widehat{\delta}}{2}>0 \quad \forall n \geqslant n_{1} \geqslant n_{0}
$$

(recall that $t_{n} \rightarrow 0$ and so $\left(h_{t_{n}}\right)^{\prime} \rightarrow \varphi^{\prime}$ ). Combining this estimate with (4.43), we reach a contradiction. This proves the Claim.

From Proposition 3.5, we know that $\varphi=h_{0}$ satisfies the Cerami condition. In a similar way, exploiting the orthogonality of the component spaces, we can check that $h_{1}$ also satisfies the Cerami condition. So, we can apply Proposition 2.6 and infer that

$$
C_{k}(\varphi, \infty)=C_{k}\left(h_{1}, \infty\right) \quad \forall k \geqslant 0 .
$$

Invoking Theorem 2.3 of $\mathrm{Li}$ and Zou [26], we have

$$
C_{k}\left(h_{1}, \infty\right)=\delta_{k, d_{i}} \mathbb{Z} \quad \forall k \geqslant 0,
$$

with $d_{i}=\operatorname{dim} \bar{H}_{i-1}$.

Now we are ready to produce the nontrivial smooth solutions of problem (1.1). We start with the solutions of constant sign.

\section{Solutions of constant sign}

First using truncations and the direct method, we produce two constant sign smooth solutions for problem (1.1) (one positive and the other negative). 
Proposition 5.1 If hypotheses $H_{f}$ (i), (iii), and (iv) hold, then problem (1.1) has two nontrivial smooth solutions of constant sign

$$
u_{0} \in \operatorname{int} C_{+}, \quad v_{0} \in-\operatorname{int} C_{+}, \quad a_{-}<v_{0}(z)<0<u_{0}(z)<a_{-} \forall z \in \bar{\Omega}
$$

and both $u_{0}, v_{0}$ are local minimizers of $\varphi$.

Proof We introduce the following truncation-perturbation of $f(z, \zeta)$ :

$$
g_{+}(z, \zeta)= \begin{cases}0 & \text { if } \zeta<0 \\ f(z, \zeta)+\zeta & \text { if } 0 \leqslant \zeta \leqslant a_{+} \\ f\left(z, a_{+}\right)+a_{+} & \text {if } a_{+}<\zeta\end{cases}
$$

Here, $a_{+}>0$ is as in hypothesis $H_{f}(i v)$. Let

$$
G_{+}(z, \zeta)=\int_{0}^{\zeta} g_{+}(z, s) \mathrm{d} s
$$

and let $\psi_{+}: H_{n}^{1}(\Omega) \longrightarrow \mathbb{R}$ be the $C^{1}$-functional, defined by

$$
\psi_{+}(u)=\frac{1}{2}\|\nabla u\|_{2}^{2}+\frac{1}{2}\|u\|_{2}^{2}-\int_{\Omega} G_{+}(z, u(z)) \mathrm{d} z \quad \forall u \in H_{n}^{1}(\Omega) .
$$

It is clear from (5.1) that $\psi_{+}$is coercive. Also, it is sequentially weakly lower semicontinuous. Invoking the Weierstrass theorem, we can find $u_{0} \in H_{n}^{1}(\Omega)$, such that

$$
\psi_{+}\left(u_{0}\right)=\inf _{u \in H_{n}^{1}(\Omega)} \psi_{+}(u)=m_{+} .
$$

Hypothesis $H_{f}($ iii $)$ implies that for a given $\varepsilon>0$, we can find $\delta=\delta(\varepsilon) \in\left(0, a_{+}\right)$, such that

$$
f(z, \zeta) \geqslant\left(\widehat{\lambda}_{m}-\varepsilon\right) \zeta \text { for almost all } z \in \Omega, \zeta \in[0, \delta],
$$

so

$$
F(z, \zeta) \geqslant \frac{1}{2}\left(\widehat{\lambda}_{m}-\varepsilon\right) \zeta^{2} \text { for almost all } z \in \Omega, \zeta \in[0, \delta] .
$$

Let $\xi \in(0, \delta]$. Then

$$
\psi_{+}(\xi)=-\int_{\Omega} F(z, \zeta) \mathrm{d} z \leqslant-\frac{1}{2}\left(\widehat{\lambda}_{m}-\varepsilon\right) \xi^{2}|\Omega|_{N}<0
$$

by choosing $\varepsilon \in\left(0, \widehat{\lambda}_{m}\right)$ (see $(5.1),(5.3)$ and recall that $\left.m \geqslant 1\right)$. So

$$
\psi_{+}\left(u_{0}\right)=m_{+}<0=\psi_{+}(0)
$$

(see (5.2)) and thus $u_{0} \neq 0$. From (5.2), we have

$$
\psi_{+}^{\prime}\left(u_{0}\right)=0
$$

so

$$
A\left(u_{0}\right)+u_{0}=N_{g_{+}}\left(u_{0}\right)
$$

where

$$
N_{g_{+}}(u)(\cdot)=g_{+}(\cdot, u(\cdot)) \quad \forall u \in H_{n}^{1}(\Omega)
$$


and thus

$$
\left\{\begin{array}{l}
-\Delta u_{0}(z)+u_{0}(z)=g_{+}(z, u(z)) \text { in } \Omega, \\
\frac{\partial u_{0}}{\partial n}=0 \text { on } \partial \Omega .
\end{array}\right.
$$

Regularity theory implies that $u_{0} \in C_{n}^{1}(\bar{\Omega}) \backslash\{0\}$. On (5.4), we act with $\left(u_{0}-a_{+}\right)^{+} \in H_{n}^{1}(\Omega)$. Then

$$
\begin{aligned}
\left\langle A\left(u_{0}\right),\left(u_{0}-a_{+}\right)^{+}\right\rangle+\int_{\Omega} u_{0}\left(u_{0}-a_{+}\right)^{+} \mathrm{d} z & =\int_{\Omega} g_{+}\left(z, u_{0}\right)\left(u_{0}-a_{+}\right)^{+} \mathrm{d} z \\
& =\int_{\Omega}\left(f\left(z, a_{+}\right)+a_{+}\right)\left(u_{0}+a_{+}\right)^{+} \mathrm{d} z
\end{aligned}
$$

(see (5.1)), so

$$
\left\langle A\left(u_{0}\right)-A\left(a_{+}\right),\left(u_{0}-a_{+}\right)^{+}\right\rangle+\int_{\Omega}\left(u_{0}-a_{+}\right)\left(u_{0}+a_{+}\right)^{+} \mathrm{d} z \leqslant 0
$$

(see hypothesis $\left.H_{f}(i v)\right)$ and thus

$$
\left|\left\{u_{0}>a_{+}\right\}\right|_{N}=0,
$$

i.e., $u_{0} \leqslant a_{+}$. Also, on (5.4), we act with $-u_{0}^{-} \in H_{n}^{1}(\Omega)$ and obtain

$$
\left\|\nabla u_{0}^{-}\right\|_{2}^{2}+\left\|u_{0}^{-}\right\|_{2}^{2}=0
$$

(see (5.1)), so $u_{0} \in C_{+} \backslash\{0\}$. Then, (5.5) becomes

$$
\left\{\begin{array}{l}
-\Delta u_{0}(z)=f\left(z, u_{0}(z)\right) \text { in } \Omega, \\
\frac{\partial u_{0}}{\partial n}=0 \text { on } \partial \Omega .
\end{array}\right.
$$

By the mean value theorem, we can find $\mu_{0}>0$, such that for almost all $z \in \Omega$, the function $\zeta \longmapsto f(z, \zeta)+\mu_{0} \zeta$ is nondecreasing on $\left[a_{-}, a_{+}\right]$. Hence,

$$
-\Delta u_{0}(z)+\mu_{0} u_{0}(z) \geqslant 0 \text { almost everywhere in } \Omega,
$$

so

$$
\Delta u_{0}(z) \leqslant \mu_{0} u_{0}(z) \text { almost everywhere in } \Omega
$$

and thus

$$
u_{0} \in \operatorname{int} C_{+}
$$

(see Vázquez [36]). Also, we have

$$
-\Delta a_{+}+\mu_{0} a_{+}=\mu_{0} a_{+} \geqslant f\left(z, a_{+}\right)+\mu_{0} a_{+} \text {almost everywhere in } \Omega
$$

(see $\left.H_{f}(i v)\right)$ and

$$
\Delta u_{0}(z)-\mu_{0} u_{0}(z)=-f\left(z, u_{0}(z)\right)-\mu_{0} u_{0}(z) \text { almost everywhere in } \Omega .
$$

Adding, we obtain

$$
-\Delta\left(a_{+}-u_{0}\right)(z)+\mu_{0}\left(a_{+}-u_{0}\right)(z) \geqslant 0 \text { almost everywhere in } \Omega,
$$

so

$$
\Delta\left(a_{+}-u_{0}\right)(z) \leqslant \mu_{0}\left(a_{+}-u_{0}\right)(z) \text { almost everywhere in } \Omega,
$$


and thus

$$
a_{+}-u_{0} \in \operatorname{int} C_{+}
$$

(see Vázquez [36]).

So, if

$$
\left[0, a_{+}\right]=\left\{u \in H_{n}^{1}(\Omega): 0 \leqslant u(z) \leqslant a_{+} \text {almost everywhere in } \Omega\right\},
$$

then from (5.6) and (5.7), it follows that $u_{0} \in$ int $C_{n}^{1}(\bar{\Omega})\left[0, a_{+}\right]$. Since $\left.\varphi\right|_{\left[0, a_{+}\right]}=\left.\psi\right|_{\left[0, a_{+}\right]}$(see (5.1)), it follows that $u_{0}$ is a local $C_{n}^{1}(\bar{\Omega})$-minimizer of $\varphi$ and so Proposition 2.2 implies that $u_{0}$ is a local $H_{n}^{1}(\Omega)$-minimizer of $\varphi$.

Similarly, truncating the function $\zeta \longmapsto f(z, \zeta)+\zeta$ at $\left\{a_{-}, 0\right\}$ and working as above, we obtain another constant sign smooth solution of (1.1):

$$
v_{0} \in-\operatorname{int} C_{+}, \quad a_{-}<v_{0}(z)<0 \quad \forall z \in \bar{\Omega}
$$

(i.e., $v_{0} \in \operatorname{int}_{C_{n}^{1}(\bar{\Omega})}\left[a_{-}, 0\right]$ ), which is a local minimizer of $\varphi$.

Let us recall the notions of upper and lower solutions for problem (1.1).

Definition 5.2 (a) We say that $\bar{u} \in H^{1}(\Omega)$ is an "upper solution" for (5.2), if

$$
\int_{\Omega}(\nabla \bar{u}, \nabla h)_{\mathbb{R}^{N}} \mathrm{~d} z \geqslant \int_{\Omega} f(z, \bar{u}) h \mathrm{~d} z \quad \forall h \in H^{1}(\Omega), h \geqslant 0 .
$$

(b) We say that $\underline{u} \in H^{1}(\Omega)$ is a "lower solution" for (5.2), if

$$
\int_{\Omega}(\nabla \bar{u}, \nabla h)_{\mathbb{R}^{N}} \mathrm{~d} z \leqslant \int_{\Omega} f(z, \bar{u}) h \mathrm{~d} z \quad \forall h \in H^{1}(\Omega), h \geqslant 0 .
$$

The next lemma is taken from Aizicovici et al. [2], where it was proved in the more general context of equations driven by the Neumann $p$-Laplacian.

Lemma 5.3 (a) If $\underline{u}_{1}, \underline{u}_{2} \in H^{1}(\Omega)$ are two lower solutions for problem (5.2), then $\underline{u}=\max \left\{\underline{u}_{1}, \underline{u}_{2}\right\} \in H^{1}(\Omega)$ is a lower solution too.

(b) If $\bar{u}_{1}, \bar{u}_{2} \in H^{1}(\Omega)$ are two upper solutions for problem (5.2), then $\bar{u}=\min \left\{\bar{u}_{1}, \bar{u}_{2}\right\} \in$ $H^{1}(\Omega)$ is an upper solution too.

Using this lemma, we can produce the biggest solution of (1.1) in the order interval $\left[0, a_{+}\right]$ and the smallest solution of $(1.1)$ in the order interval $\left[a_{-}, 0\right]$.

Proposition 5.4 If hypotheses $H_{h}(\mathrm{i})$, (iii), and (iv) hold, then problem rm (1.1) has a biggest solution $u^{*} \in \operatorname{int} C_{+}$in the order interval $\left[0, a_{+}\right]$and a smallest solution $v^{*} \in-\operatorname{int} C_{+}$in the order interval $\left[a_{-}, 0\right]$.

Proof We introduce the set

$$
\mathcal{D}_{+}=\left\{u \in H_{n}^{1}(\Omega): u \text { is a nontrivial solution of (1.1), } u \in\left[0, a_{+}\right]\right\} .
$$

From Proposition 5.1, we have that $\mathcal{D}_{+} \neq \varnothing$ and $\mathcal{D}_{+} \subseteq$ int $C_{+}$. We show that $\mathcal{D}_{+}$is upward directed, i.e., if $u_{1}, u_{2} \in \mathcal{D}_{+}$, then we can find $u \in \mathcal{D}_{+}$, such that $\max \left\{u_{1}, u_{2}\right\} \leqslant u$. To this end, let $u_{1}, u_{2} \in \mathcal{D}_{+}$and let us set $\underline{u}=\max \left\{u_{1}, u_{2}\right\} \in H_{n}^{1}(\Omega)$. Lemma 5.3(a) implies 
that $\underline{u}$ is a lower solution for problem (1.1). On the other hand, hypothesis $H_{f}(i v)$ implies that $\bar{u} \equiv a_{+}$is an upper solution for problem (1.1). Truncation $f(z, \cdot)$ at $\left\{\underline{u}(z), a_{+}\right\}$and reasoning as in Aizicovici et al. [2] (see the proof of Proposition 8), via the direct method, we produce $\widehat{u}_{0} \in\left[\underline{u}, \bar{u} \equiv a_{+}\right] \cap$ int $C_{+}$, a solution of (1.1). Then, $u_{1}, u_{2} \leqslant \widehat{u}_{0}, \widehat{u}_{0} \in \mathcal{D}_{+}$, and so we conclude that $\mathcal{D}_{+}$is upward directed.

Next we show that $\mathcal{D}_{+}$has a maximal element for the usual pointwise ordering. So, let $C \subseteq \mathcal{D}_{+}$be a chain (i.e., a totally ordered subset of $\mathcal{D}_{+}$). From Dunford and Schwartz [9, p. 336], we know that we can find a sequence $\left\{u_{n}\right\}_{n \geqslant 1} \subseteq C$, such that

$$
\sup C=\sup _{n \geqslant 1} u_{n}
$$

We have

$$
A\left(u_{n}\right)=N_{f}\left(u_{n}\right) \quad \forall n \geqslant 1,
$$

so

$$
\left\|\nabla u_{n}\right\|_{2}^{2} \leqslant c_{21} \quad \forall n \geqslant 1
$$

for some $c_{21}>0$ (recall that $u_{n} \in\left[0, a_{+}\right]$) and thus the sequence $\left\{u_{n}\right\}_{n \geqslant 1} \subseteq H_{n}^{1}(\Omega)$ is bounded.

So, we may assume that

$$
\begin{array}{ll}
u_{n} \stackrel{w}{\longrightarrow} u \quad \text { in } H_{n}^{1}(\Omega) \\
u_{n} \longrightarrow u \quad \text { in } L^{2}(\Omega) .
\end{array}
$$

If in (5.8) we pass to the limit as $n \rightarrow+\infty$ and we use (5.9), then

$$
A(u)=N_{f}(u),
$$

so $u \in \mathcal{D}_{+}$and $u=\sup C$.

Hence invoking the Kuratowski-Zorn lemma, we infer that the set $\mathcal{D}_{+}$has a maximal element $u^{*} \in \mathcal{D}_{+}$. We show that this is the desired biggest solution of (1.1) in [0, $\left.a_{+}\right]$. Indeed, let $u \in \mathcal{D}_{+}$, since $\mathcal{D}_{+}$is upward directed, we can find $\widehat{u} \in \mathcal{D}_{+}$, such that $u, u^{*} \leqslant \widehat{u}$ and the maximality of $u^{*}$ implies that $\widehat{u}=u^{*}$, hence $u \leqslant u^{*}$. Since $u \in \mathcal{D}_{+}$was arbitrary, we conclude that $u^{*}$ is the biggest solution of (1.1) in $\left[0, a_{+}\right]$.

Similarly, if we introduce the set

$$
\mathcal{D}_{-}=\left\{v \in H_{n}^{1}(\Omega): v \text { is a nontrivial solution of (1.1), } v \in\left[a_{-}, 0\right]\right\},
$$

then working as above and using this time Lemma 5.3(b), we obtain $v^{*} \in-$ int $C_{+}$, a smallest solution of (1.1) in $\left[a_{-}, 0\right]$.

In the next section, using Proposition 5.4 together with truncation techniques, combined with critical point theory and Morse theory (critical groups), we prove the complete multiplicity theorem for problem (1.1).

\section{Multiplicity theorem}

For the complete multiplicity theorem, we use the full set of hypotheses $H_{f}$. 
Theorem 6.1 If hypotheses $H_{f}$ hold, then problem (1.1) has at least five nontrivial smooth solutions:

$$
\begin{aligned}
& u_{0}, \widehat{u} \in \operatorname{int} C_{+}, \widehat{u}-u_{0} \in \operatorname{int} C_{+}, \quad u_{0}(z)<a_{+} \quad \forall z \in \bar{\Omega}, \\
& v_{0}, \widehat{v} \in \operatorname{int} C_{+}, \quad v_{0}-\widehat{v} \in \operatorname{int} C_{+}, \quad a_{-}<v_{0}(z) \quad \forall z \in \bar{\Omega}
\end{aligned}
$$

and

$$
y_{0} \in C_{n}^{1}(\bar{\Omega}) \backslash\{0\} .
$$

Proof From Proposition 5.1 we already have two nontrivial smooth solutions of constant sign

$$
u_{0} \in \operatorname{int} C_{+}, \quad v_{0} \in-\operatorname{int} C_{+}, \quad a_{-}<v_{0}(z)<0<u_{0}(z)<a_{-} \quad \forall z \in \bar{\Omega} .
$$

By virtue of Proposition 5.4, we may assume that $u_{0} \in \operatorname{int} C_{+}$is the biggest solution of (1.1) in the interval $\left[0, a_{+}\right]$and $v_{0} \in-$ int $C_{+}$is the smallest solution of (1.1) in the interval $\left[a_{-}, 0\right]$. We consider the following truncation-perturbation of $f(z, \cdot)$ :

$$
h_{+}(z, \zeta)=\left\{\begin{array}{lr}
f\left(z, u_{0}(z)\right)+u_{0}(z) & \text { if } \zeta \leqslant u_{0}(z), \\
f(z, \zeta)+\zeta & \text { if } \quad u_{0}(z)<\zeta
\end{array}\right.
$$

We set

$$
H_{+}(z, \zeta)=\int_{0}^{\zeta} h_{+}(z, s) \mathrm{d} s
$$

and consider the $C^{1}$-functional $\widetilde{\psi}_{+}: H_{n}^{1}(\Omega) \longrightarrow \mathbb{R}$, defined by

$$
\widetilde{\psi}_{+}(u)=\frac{1}{2}\|\nabla u\|_{2}^{2}+\frac{1}{2}\|u\|_{2}^{2}-\int_{\Omega} H_{+}(z, u(z)) \mathrm{d} z \quad \forall u \in H_{n}^{1}(\Omega) .
$$

Reasoning as in the proof of Proposition 3.5, we show that $\widetilde{\psi}_{+}$satisfies the Cerami condition.

Claim $3 u_{0} \in \operatorname{int} C_{+}$is a local minimizer of $\widetilde{\psi}_{+}$.

To show the Claim, we consider the following truncation of $H_{+}(z, \cdot)$ :

$$
\widehat{h}_{+}(z, \zeta)= \begin{cases}h_{+}(z, \zeta) & \text { if } \zeta<a_{+} \\ h_{+}\left(z, a_{+}\right) & \text {if } a_{+} \leqslant \zeta\end{cases}
$$

We set

$$
\widehat{H}_{+}(z, \zeta)=\int_{0}^{\zeta} \widehat{h}_{+}(z, s) \mathrm{d} s
$$

and consider the $C^{1}$-functional $\widehat{\psi}_{+}: H_{n}^{1}(\Omega) \longrightarrow \mathbb{R}$, defined by

$$
\widehat{\psi}_{+}(u)=\frac{1}{2}\|\nabla u\|_{2}^{2}+\frac{1}{2}\|u\|_{2}^{2}-\int_{\Omega} \widehat{H}_{+}(z, u(z)) \mathrm{d} z \quad \forall u \in H_{n}^{1}(\Omega) .
$$

By virtue of (6.3), $\widehat{\psi}_{+}$is coercive, and of course, it is sequentially weakly lower semicontinuous. So, we can find $\widehat{u}_{0} \in H_{0}^{1}(\Omega)$, such that

$$
\widehat{\psi}_{+}\left(\widehat{u}_{0}\right)=\inf _{u \in H_{n}^{1}(\Omega)} \widehat{\psi}_{+}(u)=\widehat{m}_{+},
$$


so $\widehat{\psi}_{+}^{\prime}\left(\widehat{u}_{0}\right)=0$ and thus

$$
A\left(\widehat{u}_{0}\right)+\widehat{u}_{0}=N_{\widehat{h}_{+}}\left(\widehat{u}_{0}\right),
$$

where

$$
N_{\widehat{h}_{+}}(u)(\cdot)=\widehat{h}_{+}(\cdot, u(\cdot)) \quad \forall u \in H_{n}^{1}(\Omega) .
$$

On (6.4), we act with $\left(u_{0}-\widehat{u}_{0}\right)^{+} \in H_{n}^{1}(\Omega)$. Then

$$
\begin{aligned}
\int_{\left\{u_{0}>\widehat{u}_{0}\right\}} & \left(\nabla \widehat{u}_{0}, \nabla u_{0}-\nabla \widehat{u}_{0}\right)_{\mathbb{R}^{N}} \mathrm{~d} z+\int_{\left\{u_{0}>\widehat{u}_{0}\right\}} \widehat{u}_{0}\left(u_{0}-\widehat{u}_{0}\right) \mathrm{d} z \\
= & \int_{\left\{u_{0}>\widehat{u}_{0}\right\}} f\left(z, u_{0}\right)\left(u_{0}-\widehat{u}_{0}\right) \mathrm{d} z+\int_{\left\{u_{0}>\widehat{u}_{0}\right\}} u_{0}\left(u_{0}-\widehat{u}_{0}\right) \mathrm{d} z \\
= & \int_{\left\{u_{0}>\widehat{u}_{0}\right\}}\left(\nabla u_{0}, \nabla u_{0}-\nabla \widehat{u}_{0}\right) \mathrm{d} z+\int_{\left\{u_{0}>\widehat{u}_{0}\right\}} u_{0}\left(u_{0}-\widehat{u}_{0}\right) \mathrm{d} z,
\end{aligned}
$$

so

$$
\left\|\left(u_{0}-\widehat{u}_{0}\right)^{+}\right\|^{2}=0
$$

and thus

$$
u_{0} \leqslant \widehat{u}_{0} .
$$

Also, acting on (6.4) with $\left(\widehat{u}_{0}-a_{+}\right)^{+} \in H_{n}^{1}(\Omega)$, we obtain

$$
\begin{array}{r}
\int_{\left\{\widehat{u}_{0}>a_{+}\right\}}\left\|\nabla \widehat{u}_{0}\right\|^{2} \mathrm{~d} z+\int_{\left\{\widehat{u}_{0}>a_{+}\right\}} \widehat{u}_{0}\left(\widehat{u}_{0}-a_{+}\right) \mathrm{d} z \\
=\int_{\left\{\widehat{u}_{0}>a_{+}\right\}}\left(f\left(z, a_{+}\right)+a_{+}\right)\left(\widehat{u}_{0}-a_{+}\right) \mathrm{d} z
\end{array}
$$

(see (6.2) and (6.3)), so

$$
\left\|\left(u_{0}-a_{+}\right)^{+}\right\|^{2}=0
$$

and thus

$$
u_{0} \leqslant a_{+}
$$

From (6.5) and (6.6), it follows that $\widehat{u}_{0} \in\left[u_{0}, a_{+}\right]$and (6.4) becomes

$$
A\left(\widehat{u}_{0}\right)=N_{f}\left(\widehat{u}_{0}\right)
$$

(see (6.2) and (6.3)), so

$$
\left\{\begin{array}{l}
-\Delta \widehat{u}_{0}(z)=f\left(z, \widehat{u}_{0}(z)\right) \text { in } \Omega, \\
\frac{\partial \widehat{u}_{0}}{\partial n}=0 \text { on } \partial \Omega
\end{array}\right.
$$

and thus $\widehat{u}_{0} \in \operatorname{int} C_{+}$(regularity theory) solves (1.1) and $u_{0} \in\left[u_{0}, a_{+}\right]$.

But recall that $u_{0} \in$ int $C_{+}$is the biggest solution of (1.1) in [0, $\left.a_{+}\right]$. Hence, $\widehat{u}_{0}=u_{0}$.

Since $\left.\widetilde{\psi}_{+}\right|_{\left[0, a_{+}\right]}=\left.\widehat{\psi}_{+}\right|_{\left[0, a_{+}\right]}\left(\operatorname{see}(6.2)\right.$ and (6.3)) and $u_{0} \in \operatorname{int}_{C_{n}^{1}(\bar{\Omega})}\left[0, a_{+}\right]$(see (6.1)), it follows that $u_{0}=\widehat{u}_{0}$ is a local $C_{n}^{1}(\bar{\Omega})$-minimizer of $\widetilde{\psi}_{+}$; hence by Proposition 2.2, it is also a local $H_{n}^{1}(\Omega)$-minimizer of $\widetilde{\psi}_{+}$. This proves the Claim. 
We may assume that $u_{0}$ is an isolated critical point of $\widetilde{\psi}_{+}$. To see this, suppose that we can find $\left\{u_{n}\right\}_{n} \geqslant 1 \subseteq H_{n}^{1}(\Omega)$, such that

$$
u_{n} \longrightarrow u_{0} \text { in } H_{n}^{1}(\Omega) \text { and } \widetilde{\psi}_{+}^{\prime}\left(u_{n}\right)=0 \quad \forall n \geqslant 1 .
$$

Then as above, we show that $u_{n} \geqslant u_{0}$ for all $n \geqslant 1$ and $\left\{u_{n}\right\}_{n} \geqslant 1 \subseteq$ int $C_{+}$(regularity theory). From (6.2), it follows that $\left\{u_{n}\right\}_{n} \geqslant 1$ is a whole sequence of distinct positive smooth solutions of (1.1) and so we are done. So, assuming that $u_{0}$ is an isolated critical point of $\widetilde{\psi}_{+}$and reasoning as in Aizicovici et al. [1] (see the proof of Proposition 29), we can find $\widetilde{\varrho}>0$ small, such that

$$
\widetilde{\psi}_{+}\left(u_{0}\right)<\inf \left\{\widetilde{\psi}_{+}(u):\left\|u-u_{0}\right\|=\widetilde{\varrho}\right\}=\widetilde{m}_{+} .
$$

Let $\xi>\left\|u_{0}\right\|_{\infty}$. Then

$$
\widetilde{\psi}_{+}(\xi) \leqslant c_{22}-\int_{\Omega} F(z, \xi) \mathrm{d} z,
$$

for some $c_{22}>0$ (see (6.2)). By virtue of hypothesis $H_{f}(i i)$, for a given $\varepsilon \in\left(0, \widehat{\lambda}_{i}\right)$, we can find $M>0$, such that

$$
F(z, \zeta) \geqslant \frac{\widehat{\lambda}_{i}-\varepsilon}{2} \zeta^{2} \text { for almost all } z \in \Omega, \text { all }|\zeta| \geqslant M
$$

so

$$
F(z, \zeta) \longrightarrow+\infty \text { uniformly for almost all } z \in \Omega \text { as } \zeta \rightarrow+\infty
$$

and thus

$$
\widetilde{\psi}_{+}(\xi) \longrightarrow-\infty \text { as } \xi \rightarrow+\infty
$$

(see (6.8)). From (6.7), (6.9) and since $\widetilde{\psi}_{+}$satisfies the Cerami condition, we see that we can apply the mountain pass theorem (see Theorem 2.1) and produce $\widehat{u} \in H_{n}^{1}(\Omega)$, such that

$$
\widetilde{\psi}_{+}\left(u_{0}\right)<\widetilde{m}_{+} \leqslant \widetilde{\psi}_{+}(\widehat{u})
$$

(see (6.7)) and so

$$
\widetilde{\psi}_{+}^{\prime}\left(u_{0}\right)=0 .
$$

From (6.10), we have $\widehat{u} \neq u_{0}$. From (6.11), we have

$$
A(\widehat{u})+\widehat{u}=N_{h_{+}}(\widehat{u}),
$$

where

$$
N_{h_{+}}(u)(\cdot)=h_{+}(\cdot, u(\cdot)) \quad \forall u \in H_{n}^{1}(\Omega) .
$$

Acting on (6.12) with $\left(u_{0}-\widehat{u}\right)^{+} \in H_{n}^{1}(\Omega)$ and reasoning as before, we show that $u_{0} \leqslant \widehat{u}$ and $\widehat{u} \in$ int $C_{+}$solves (1.1) (see (6.2)). Let $\widehat{\mu}>0$ be such that for almost all $z \in \Omega$, the function $\zeta \longmapsto f(z, \zeta)+\widehat{\mu} \zeta$ is nondecreasing on $\left[-\|\widehat{u}\|_{\infty},\|\widehat{u}\|_{\infty}\right]$ (it exists by the mean value theorem and hypothesis $\left.H_{f}(i)\right)$. Then

$$
-\Delta\left(\widehat{u}-u_{0}\right)(z)+\widehat{\mu}\left(\widehat{u}-u_{0}\right)(z) \geqslant 0 \text { almost everywhere on } \Omega,
$$

so

$$
\Delta\left(\widehat{u}-u_{0}\right)(z) \leqslant \widehat{\mu}\left(\widehat{u}-u_{0}\right)(z) \text { almost everywhere on } \Omega,
$$


and thus $\widehat{u}-u_{0} \in \operatorname{int} C_{+}$(see Vázquez [36]).

Similarly, if we use the negative smooth solution $v_{0} \in-$ int $C_{+}$and by considering the truncation-perturbation of $f(z, \cdot)$, defined by

$$
h_{-}(z, \zeta)= \begin{cases}f(z, \zeta)+\zeta & \text { if } \zeta<v_{0}(z), \\ f\left(z, v_{0}(z)\right)+v_{0}(z) & \text { if } v_{0}(z) \leqslant \zeta\end{cases}
$$

then working as above, we produce a second negative smooth solution $\widehat{v} \in-\operatorname{int} C_{+}$, such that $v_{0}-\widehat{v} \in \operatorname{int} C_{+}$.

Since $u_{0}, v_{0}$ are local minimizers of $\varphi$, then

$$
C_{k}\left(\varphi, u_{0}\right)=C_{k}\left(\varphi, v_{0}\right)=\delta_{k, 0} \mathbb{Z} \quad \forall k \geqslant 0 .
$$

From the previous considerations, we know that $\widehat{u} \in$ int $C_{+}$is a critical point of mountain pass type for the functional $\widetilde{\psi}_{+}$. Note that

$$
\widetilde{\psi}_{+}(\widehat{u})=\frac{1}{2}\|\nabla \widehat{u}\|_{2}^{2}-\int_{\Omega} F(z, \widehat{u}) \mathrm{d} z+\int_{\Omega}\left(F\left(z, u_{0}\right)-f\left(z, u_{0}\right) u_{0}\right) \mathrm{d} z
$$

(see (6.2)), so

$$
\widetilde{\psi}_{+}(\widehat{u})=\varphi(\widehat{u})+c_{23},
$$

with $c_{23}=\int_{\Omega}\left(F\left(z, u_{0}\right)-f\left(z, u_{0}\right) u_{0}\right) \mathrm{d} z$. Thus

$$
C_{k}\left(\widetilde{\psi}_{+}, \widehat{u}\right)=C_{k}(\varphi, \widehat{u}) \quad \forall k \geqslant 0 .
$$

Since $\widehat{u}$ is a critical point of mountain pass type for $\widetilde{\psi}_{+}$, it follows that

$$
C_{1}\left(\widetilde{\psi}_{+}, \widehat{u}\right) \neq 0
$$

(see e.g., [7, p. 69]). So

$$
C_{1}(\varphi, \widehat{u}) \neq 0
$$

(see (6.14)).

Recall that $\varphi \in C^{2}\left(H_{n}^{1}(\Omega)\right)$ and $\varphi^{\prime \prime}(\widehat{u})$ is a Fredholm operator. Moreover, if $\sigma\left(\varphi^{\prime \prime}(\widehat{u})\right) \subseteq$ $[0,+\infty)$, then $\operatorname{ker} \varphi^{\prime \prime}(\widehat{u})=\{0\}$ if $0 \notin \sigma\left(\varphi^{\prime \prime}(\widehat{u})\right)$ and $\operatorname{dim} \operatorname{ker} \varphi^{\prime \prime}(\widehat{u})=1$ if $0 \in \sigma\left(\varphi^{\prime \prime}(\widehat{u})\right)$ (see Sect. 2). This fact together with (6.15) permits the use of Proposition 2.5 of Bartsch [4] and so we have

$$
C_{k}(\varphi, \widehat{u})=\delta_{k, 1} \mathbb{Z} \quad \forall k \geqslant 0
$$

Similarly, we show that

$$
C_{k}(\varphi, \widehat{v})=\delta_{k, 1} \mathbb{Z} \quad \forall k \geqslant 0 .
$$

From Proposition 4.3, we know that

$$
C_{k}(\varphi, 0)=\delta_{k, d_{m}} \mathbb{Z} \quad \forall k \geqslant 0 .
$$

From Proposition 4.4, we know that

$$
C_{k}(\varphi, \infty)=\delta_{k, d_{i}} \mathbb{Z} \quad \forall k \geqslant 0 .
$$

This implies that there exists $y_{0} \in K_{\varphi}$, such that $C_{d_{i}}\left(\varphi, y_{0}\right) \neq 0$ (see (2.1)). Since $i \geqslant 1$ (see hypothesis $H_{f}(i i)$ ), it follows that $d_{i} \geqslant 2$ and $d_{i} \neq d_{m}$ since $i \neq m$ (see $H_{f}(i i)$ ). So, from this fact and (6.13), (6.16)-(6.18), we conclude that $y_{0} \notin\left\{0, u_{0}, v_{0}, \widehat{u}, \widehat{v}\right\}$. Hence, $y_{0} \in C_{n}^{1}(\bar{\Omega}) \backslash\{0\}$ (regularity theory) is a fifth nontrivial smooth solution of (1.1). 
Acknowledgments The authors would like to thank the referee for their corrections and remarks.

Open Access This article is distributed under the terms of the Creative Commons Attribution Noncommercial License which permits any noncommercial use, distribution, and reproduction in any medium, provided the original author(s) and source are credited.

\section{References}

1. Aizicovici, S., Papageorgiou, N.S., Staicu, V.: Degree theory for operators of monotone type and nonlinear elliptic equations with inequality constraints. Mem. Am. Math. Soc. 196(915) (2008)

2. Aizicovici, S., Papageorgiou, N.S., Staicu, V.: Existence of multiple solutions with precise sign information for superlinear Neumann problems. Ann. Mat. Pura Appl. 188(4), 679-719 (2009)

3. Bartolo, P., Benci, V., Fortunato, D.: Abstract critical point theorems and applications to some nonlinear problems with "strong" resonance at infinity. Nonlinear Anal. 7, 981-1012 (1983)

4. Bartsch, T.: Critical point theory on partially ordered Hilbert spaces. J. Funct. Anal. 186, 117-152 (2001)

5. Bartsch, T., Li, S.-J.: Critical point theory for asymptotically quadratic functionals and applications to problems with resonance. Nonlinear Anal. 28, 419-441 (1997)

6. Brézis, H., Nirenberg, L.: $H^{1}$ versus $C^{1}$ local minimizers. C. R. Acad. Sci. Paris Sér. I Math. 317, 465-472 (1993)

7. Chang, K.-C.: Infinite-Dimensional Morse Theory and Multiple Solution Problems, vol. 6 of Progress in Nonlinear Differential Equations and Their Applications. Birkhäuser Verlag, Boston (1993)

8. Costa, D.G., Silva, E.A.B.: On a class of resonant problems at higher eigenvalues. Diff. Integral Equ. 8, 663-671 (1995)

9. Dunford, N., Schwartz, J.T.: Linear Operators, I General Theory, vol. 7 of Pure and Applied Mathematics. Wiley, New York (1958)

10. Filippakis, M., Papageorgiou, N.S.: Multiple nontrivial solutions for resonant Neumann problems. Math. Nachr. 283, 1000-1014 (2010)

11. García Azorero, J., Manfredi, J., Peral Alonso, I.: Sobolev versus Hölder local minimizers and global multiplicity for some quasilinear elliptic equations. Commun. Contemp. Math. 2, 385-404 (2000)

12. Gasiński, L., Papageorgiou, N.S.: Nonlinear Analysis. Chapman and Hall/CRC Press, Boca Raton (2006)

13. Guo, Z., Zhang, Z.: $W^{1, p}$ versus $C^{1}$ local minimizers and multiplicity results for quasilinear elliptic equations. J. Math. Anal. Appl. 286, 32-50 (2003)

14. Hirano, N., Nishimura, T.: Multiplicity results for semilinear elliptic problems at resonance with jumping nonlinearities. J. Math. Anal. Appl. 180, 566-586 (1993)

15. Iannacci, R., Nkashama, M.N.: Nonlinear two point boundary value problems at resonance without Landesman-Lazer condition. Proc. Am. Math. Soc. 106, 943-952 (1989)

16. Iannacci, R., Nkashama, M.N.: Nonlinear elliptic partial differential equations at resonance: higher eigenvalues. Nonlinear Anal. 25, 455-471 (1995)

17. Iannizzotto, A., Papageorgiou, N.S.: Existence of three nontrivial solutions for nonlinear Neumann hemivariational inequalities. Nonlinear Anal. 70, 3285-3297 (2009)

18. Kristaly, A., Papageorgiou, N.S.: Multiple nontrivial solutions for neumann problems involving the p-Laplacian: a Morse theoretic approach. Adv. Nonlinear Stud. 124, 83-87 (1996)

19. Kuo, C.-C.: On the solvability of a nonlinear second order elliptic equations at resonance. Proc. Am. Math. Soc. 124, 83-87 (1996)

20. Landesman, E.M., Robinson, S.B., Rumbos, A.: Multiple solutions of semilinear elliptic problems at resonance. Nonlinear Anal. 24, 1049-1059 (1995)

21. Li, C.: The existence of infinitely many solutions of a class of nonlinear elliptic equations with Neumann boundary condition for both resonance and oscillation problems. Nonlinear Anal. 54, 431-443 (2003)

22. Liang, Z., Su, J.: Multiple solutions for semilinear elliptic boundary value problems with double resonance. J. Math. Anal. Appl. 354, 147-158 (2009)

23. Li, C., Li, S.: Multiple solutions and sign-changing solutions of a class of nonlinear elliptic equations with Neumann boundary condition. J. Math. Anal. Appl. 298, 14-32 (2004)

24. Liu, S.-B.: Nontrivial solutions for elliptic problems. Nonlinear Anal. 70, 1965-1974 (2009)

25. Li, S.-J., Su, J.: Existence of multiple solutions of a two-point boundary value problems at resonance. Topol. Methods Nonlinear Anal. 10, 123-135 (1997)

26. Li, S.-J., Zou, W.: The computations of the critical groups with an applications to elliptic resonant problems at a higher eigenvalues. J. Math. Anal. Appl. 235, 237-259 (1999) 
27. Mawhin, J.: Semicoercive monotone variational problems. Acad. Roy. Belg. Bull. Cl. Sci. 73(5), 118-130 (1987)

28. Mawhin, J., Ward, J., Willem, M.: Variational methods and semi-linear elliptic equations. Arch. Ration. Mech. Anal. 95, 269-277 (1986)

29. Mawhin, J., Willem, M.: Critical Point Theory and Hamiltonian Systems, vol. 74 of Applied Mathematical Sciences. Springer, New York (1989)

30. Motreanu, D., Motreanu, V.V., Papageorgiou, N.S.: Nonlinear Neumann problems near resonance. Indiana Univ. Math. J. 58, 1257-1279 (2009)

31. Motreanu, D., Papageorgiou, N.S.: Multiple solutions for nonlinear Neumann problems driven by a nonhomogeneous differential operators. Proc. Am. Math. Soc. (to appear)

32. Perera, K., Schechter, M.: Solutions of nonlinear equations having asymptotic limits at zero and infinity. Calc. Var. Partial Diff. Equ. 12, 359-369 (2001)

33. Qian, A.: Existence of infinitely many solutions for a superlinear Neumann boundary value problem. Boundary Value Probl. 2005, 329-335 (2005)

34. Su, J., Tang, C.-L.: Multiplicity results for semilinear elliptic equations with resonance at higher eigenvalues. Nonlinear Anal. 44, 311-321 (2001)

35. Tang, C.-L., Wu, X.-P.: Existence and multiplicity for solutions of Neumann problems for elliptic equations. J. Math. Anal. Appl. 288, 660-670 (2003)

36. Vázquez, J.L.: A strong maximum principle for some quasilinear elliptic equation. Appl. Math. Optim. 12, 191-202 (1984)

37. Zou, W.: Multiple solutions results for two-point boundary value problem with resonance. Discrete Contin. Dyn. Syst. 4, 485-496 (1998)

38. Zou, W., Liu, J.-Q.: Multiple solutions for resonant elliptic equations via local linking theory and Morse theory. J. Diff. Equ. 170, 68-95 (2001) 